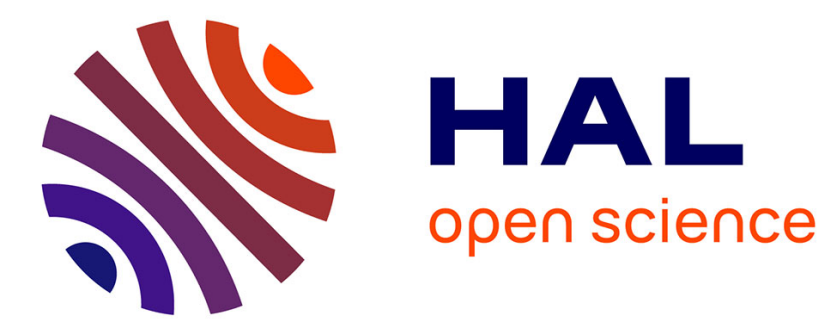

\title{
Perception et pratique de l'espace maritime par les fonctionnaires chinois (XIXe - début du XIXe siècle) \\ Paola Calanca
}

\section{To cite this version:}

Paola Calanca. Perception et pratique de l'espace maritime par les fonctionnaires chinois (XIXe

- début du XIXe siècle). Bulletin de l'Ecole française d'Extrême-Orient, 2010, 97 (1), pp.21-54.

10.3406/befeo.2010.6128. halshs-02512937

\section{HAL Id: halshs-02512937 \\ https://shs.hal.science/halshs-02512937}

Submitted on 20 Mar 2020

HAL is a multi-disciplinary open access archive for the deposit and dissemination of scientific research documents, whether they are published or not. The documents may come from teaching and research institutions in France or abroad, or from public or private research centers.
L'archive ouverte pluridisciplinaire HAL, est destinée au dépôt et à la diffusion de documents scientifiques de niveau recherche, publiés ou non, émanant des établissements d'enseignement et de recherche français ou étrangers, des laboratoires publics ou privés. 


\section{Perception et pratique de l'espace maritime par les fonctionnaires} chinois (XIXe - début du XIXe siècle)

\section{Paola Calanca}

\section{Citer ce document / Cite this document :}

Calanca Paola. Perception et pratique de l'espace maritime par les fonctionnaires chinois (XIXe - début du XIXe siècle). In: Bulletin de l'Ecole française d'Extrême-Orient. Tome 97-98, 2010. pp. 21-54;

doi : https://doi.org/10.3406/befeo.2010.6128

https://www.persee.fr/doc/befeo_0336-1519_2010_num_97_1_6128

Fichier pdf généré le 08/02/2019 


\begin{abstract}
At a time when China is displaying her maritime ambition, it is interesting to revisit the long process of expansion of its maritime spaces between the 14th and the early 19th centuries. This is a time during which the coastal line was finally integrated into the imperial security system through the establishment, at the beginning of the Ming dynasty, of a fortifications network covering the entire Chinese littoral, and the reconsideration of this organization with the irruption of European powers in the middle of the 19th century. In this paper, we will try to understand how this space was perceived by the government, depending on the period, through the analysis of the terminology used by its representatives, and the meaning of the terms neihai (inner seas) and waihai (high seas) when applied to the incorporation of certain islands into the national territory.
\end{abstract}

\begin{abstract}
Résumé
À l'heure où la Chine affiche son ambition maritime, il était important de revenir sur le long processus d'élargissement de l'espace maritime par le gouvernement durant la période comprise entre le XIVe et le début du XIXe siècle. Une époque importante car elle marque, d'une part, les prémices de l'intégration définitive du littoral au dispositif sécuritaire de l'empire, à travers l'établissement d'un système de fortifications couvrant l'ensemble de la côte à partir du début des Ming et, d'autre part, la remise en cause de cette organisation avec l'irruption des puissances européennes sur le sol chinois. II s'est ainsi agi de comprendre comment cet espace a été appréhendé par la cour et ses représentants selon les époques, en étudiant la terminologie employée pour le désigner et en analysant les acceptions de neihai (la mer intérieure) et de waihai (la haute mer), lorsque ces termes sont utilisés pour évoquer l'incorporation de certaines îles à l'empire.
\end{abstract}




\title{
Perception et pratique de l'espace maritime par les fonctionnaires chinois ( $\mathrm{XIV}^{\mathrm{e}}$-début du XIX $\mathrm{X}^{\mathrm{e}}$ siècle)
}

\author{
Paola Calanca*
}

En Chine, les confins orientaux et méridionaux (la bande littorale) ont, dans l'ensemble, moins retenu l'attention des gouvernants et des auteurs de leur époque que les limites septentrionales, où la menace d'invasion a été l'une des constantes de l'histoire dynastique. Jusqu'à un passé récent ${ }^{1}$, les travaux historiques consacrés à ce domaine ont, le plus souvent, traité la question frontalière dans le cadre du tribut et de la politique de fermeture, la fameuse «prohibition maritime » (haijin 海禁), ou encore dans celui de la modernisation manquée du pays et de ses efforts pour se doter d'une flotte de guerre digne de ce nom ${ }^{2}$. Il est vrai que les sources anciennes ne sont pas très dissertes sur ce sujet et que les données sont très dispersées. Le contraste est saisissant entre cette documentation morcelée et les ouvrages publiés depuis quelques années au sujet des frontières maritimes actuelles. Cela n'est pas surprenant, car l'appropriation des mers et des océans par des États souverains est un phénomène récent, apparu à la fin du dernier conflit mondial et surtout manifeste à partir des années 1970. Depuis, et à travers la Convention des Nations unies sur le Droit de la mer entrée en vigueur en $1994^{3}$, on assiste à un processus d'étatisation des espaces marins et d'extension des zones de souveraineté.

* Maitre de conférences à l'École française d’Extrême-Orient.

1. À la fin des années 1980, des Histoires générales ont paru qui présentent les connaissances maritimes depuis l'époque ancienne jusqu' à la fin de l’empire : rarement citées, elles n’en constituent pas moins des ouvrages de référence au sujet de la vision que les Chinois avaient des mers qui les entouraient et

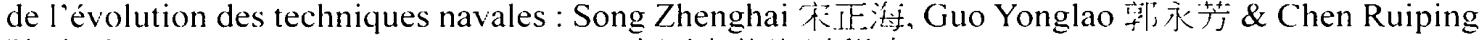

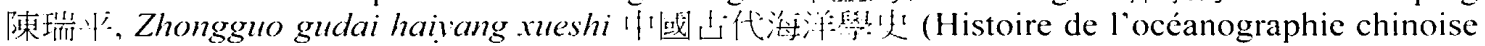
ancienne), 1989 : Sun Guangqi 䋆炕圻, Zhongguo gudai hanghai shi 中國古代航海这 (Histoire de la navigation ancienne chinoise), 1989 ; Zhang Xun (éd.), Zhongguo hanghai keji shi 中國航海科技史 (Histoire de la technique navale chinoise), 1991. À partir de 2007, la maison d'édition Haiyang de Pékin lance un vaste projet, dirigé par Sun Guangqi, avec cinq volumes déjà parus : Zhongglıo hanghai shi

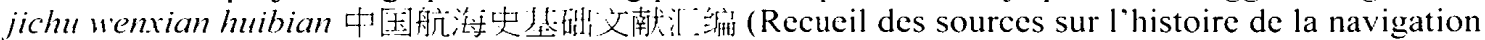
chinoise). Avec le livre sus-cité. édité par Angela Schottenhammer et Roderich Ptak, nous disposons également d'un premier recueil d'études en anglais sur la perception de l'espace maritime chinois.

2. En chinois, voir par exemple : Chen Wenshi 陳文石 1966, Chen Shangsheng 陳治勝 1993, ctc. Pour l'époque mandchoue, se reporter par exemple au travail de Wang Hongbin 王芸斌 (2002) beaucoup moins influencé par ces stéréotypes. Pour un aperçu des travaux réalisés sur ce sujet au cours des dernières décennies du $\mathrm{x}^{\mathrm{c}} \mathrm{c}^{\mathrm{e}}$ siècle, lorsque l’histoire maritime connut un renouveau en Chine, se reporter

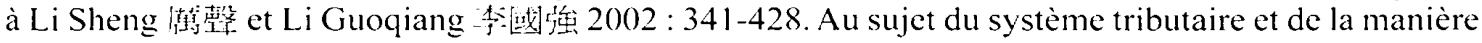
dont il a été interprété à cette époque, voir Fairbank 1969.

3. La première convention du droit de la mer remonte à la conférence de Genève de 1958 : elle fut suivie de deux autres réunions en 1962 et 1966. Une troisième fut convoquée par les Nations unies : 
La Chine ne fait pas figure d'exception : c'est au cours des dernières décennies du $\mathrm{xx}^{\mathrm{e}}$ siècle qu'elle prend toute la mesure de l'enjeu que constitue le contrôle des mers et des richesses qu'elles renferment. Elle a, depuis, adopté une politique expansionniste, une première au regard de l'apathie et de la prudence qui caractérisèrent jusque-là sa politique dans ce domaine, à l'exclusion de certaines périodes (voir infira). Pour ce faire, elle a recours à l'un des arguments les plus invoqués dans les revendications territoriales, à savoir la pratique historique de certains espaces frontaliers ${ }^{4}$, ce qui lui permet d'attester de la longue fréquentation, voire de l'installation, de ressortissants chinois sur les îles et les îlots contestés ${ }^{5}$. Or, du début des Ming (1368-1644) jusqu'après le milieu du XIX siècle, nul n'était autorisé à émigrer outre-mer et ceux qui le faisaient étaient considérés comme des hors-la-loi. Cela revient ainsi à accorder à des "rebelles " et à des "pirates » l'honneur d'avoir découvert, visité, voire peuplé, des terres que les gouvernants de l'époque n'avaient jamais songé à inscrire dans les registres cadastraux (bantu 版圖). Il ne s'agit pas de se prononcer sur le bien-fondé de ces revendications tardives, mais de comprendre comment cet espace a été appréhendé, en fonction des époques, par la cour et ses représentants ${ }^{6}$.

L'étude de la terminologie employée servira de premier indicateur, puis il s'agira d'analyser les acceptions de neihai 海 (la mer intérieure) et de waihai 外海 (la haute mer) à travers l'historique de l'incorporation de certaines îles à l'empire. Afin de mettre en valeur l'évolution lexicale des expressions se rapportant à la notion de frontière maritime, la période comprise entre la fin du XIV et la première moitié du XIX ${ }^{\complement}$ siècle a été retenue. Elle nous a semblé pertinente en ce qu'elle marque, d'une part, le début de l'intégration définitive du littoral au dispositif sécuritaire de l'empire avec l'établissement d'un système de fortifications couvrant l'ensemble de la côte à partir du début des Ming et, d'autre part, la remise en cause de cette organisation avec l'irruption des puissances européennes sur le sol chinois. Au cours de cette dernière époque, la Chine a été contrainte de reconsidérer sa politique maritime, plus généralement celle de ses confins, et d'intégrer le caractère durable de l'extension des frontières européennes en Asie. Cela n'a pas seulement impliqué une meilleure prise en compte de ces régions et la refonte des forces navales, mais également la modification des relations politiques et

elle siégea une première fois à New York en 1973 et une seconde fois à Montego Bay (Jamaïque) en 1982, où les travaux aboutirent à la signature de la convention des Nations unies sur le droit de la mer (CNUDM) qui entra en vigueur en novembre 1994.

4. Les pratiques historiques de certaines activités dans des lieux donnés constituent encore de nos jours un indicateur frontalier employé, par exemple, pour la délimitation des zones de pêche.

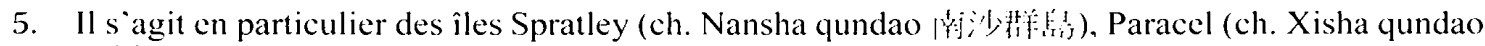

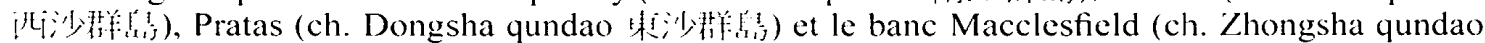

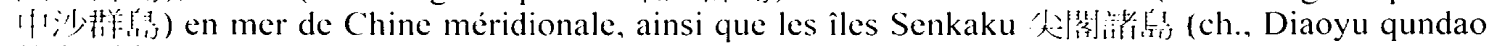

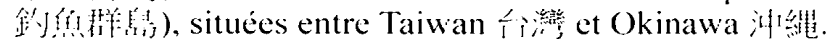

6. La première délimitation contemporaine de la frontière maritime méridionale chinoise remonterait à la carte dressće par le géographe Hu Jinjie 胡行控 (1870-1934) en 1914. Elle semble avoir inclut les îles Pratas et les Paracel : son point le plus méridional était localisé autour du $15-16^{\circ}$ de latitude Nord. En 1933, suite à l'occupation par la France de neuf îles des Spratley, cette ligne s'étendit encore vers le sud jusqu à la zone comprise entre le $7^{\circ}$ et le $9^{\circ}$ de latitude Nord. Deux ans plus tard, un document officiel y englobait aussi le récif James (ch. Zengmu ansha rigl lifliti 沙), situé autour du $4^{\circ}$ de latitude Nord, au large de Sarawak (Malaisie). Il fallut néanmoins attendre 1947 pour qu une carte officielle. publiée par le gouvernement, authentifie l'existence et l'extension de cette ligne de démarcation. dite

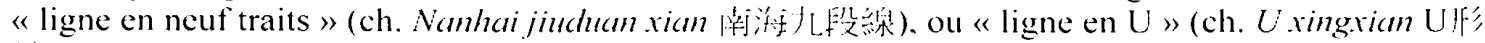
㲾), ou encore 《langue de bouf» (During laroi bo) en vietnamien (Zou Keyuan 2005 : ch. 3 ). 
économiques entretenues avec l'étranger qui s'étaient exprimées jusque-là, principalement avec les pays asiatiques, par des rapports hiérarchiques de patronage et de réciprocité dans les échanges marchands.

\section{Espaces maritimes : terminologie}

L'emploi des termes frontaliers, comme l'évolution éventuelle de leur signification, est intrinsèquement lié au contexte historique et permet de suivre le processus d'appropriation d'un espace donné. Bien que l'appréhension du milieu océanique ait requis un temps relativement lent, il en a été de même pour la nomenclature qui lui est inhérente et pour son abornement. Cela s'explique probablement par le fait que dans la cosmogonie chinoise et dans celles de maintes autres contrées, l'eau entourait la terre. Cette masse liquide était perçue comme homogène et continue, d'où une évidente difficulté à la délimiter. En revanche, son appellation, hai 海 - communément traduite de nos jours par « mer »-, se retrouve dans quelques-unes des expressions chinoises les plus utilisées pour exprimer la notion d'appartenance à un pays, à une culture ${ }^{7}$. Cette ancienne signification suggère la séparation entre deux mondes : l'un connu et ordonnancé, l'autre inconnu et indéfini. Une image finalement universelle, car " dans toutes les anciennes langues, de l'Inde à l'Irlande, le nom de mer a pour synonyme ou analogue le désert et la nuit », comme le précisait déjà au milieu du $x_{1}{ }^{e}$ siècle l'un des premiers historiens français à s'y être intéressé (Michelet 1861:43) ${ }^{8}$. Cette fonction de démarcation apparaît plus explicitement dans les locutions sihai 四海 (les quatre mers), hainei 海內 (l'intérieur des mers, le pays) et haiwai 海外 (l'étranger, les pays étrangers ; la mer extérieure). La première pourrait faire référence aux sifang 四广j (les quatre directions, ou quatre points cardinaux), soit les frontières de l'empire ${ }^{9}$. Les deux suivantes, utilisées depuis fort longtemps, distinguent la Chine (l'intérieur) des autres pays (l'extérieur).

La terminologie relative au domaine maritime se développa suivant les avancées, directes et indirectes, des Chinois loin de leurs côtes. L'extension de leur domination vers le sud pendant le premier millénaire de notre ère et l'assujettissement de populations accoutumées à la mer qui suivit, l'intensification du commerce maritime à partir du $\mathrm{VIII}^{\mathrm{e}}$ siècle, les progrès scientifiques dans le domaine de la géographie, de la cartographie, de l'astronomie et des mathématiques élargirent graduellement les connaissances des Chinois sur l'étendue océanique et permirent de déterminer progressivement les contours de certaines mers ( $\mathrm{Li}$ Xiaocong 2006:155-176). Les expéditions menées au vII siècle par les Sui (589-618) contre les Liuqiu 琉球 (Taiwan ou les Ryūkyū 琉球群島) et la Corée, puis contre ce dernier pays par les Tang (618-907), suivies à la fin du XIII' ${ }^{\mathfrak{e}}$ siècle par les opérations militaires de Kubilai (元世祖忽必烈, r. 1277-1295) à l'encontre du Japon, de la Corée, du Jiaozhi 交趾 (nord du Vietnam), de Java et de la Birmanie, enrichirent

7. Au sujet du terme hai dans les sources littéraires chinoises, voir Lanciotti $1995: 7-13$; Schottenhammer \& Ptak 2006 : 3-4.

8. L'idée de la « nuit », des « ténèbres », se retrouve également dans la définition du caractère hai donnée par l'auteur du dictionnaire Explication des noms: «Hai (mer), c'est-à-dire hui 将主 (obscurité), dont l'eau est noire comme la nuit » (Shi ming : vol. 13, p. 7b).

9. Plusieurs occurrences étayent d'ailleurs cette signification, comme la définition donnée dans le Erya 爾雅: «Les neuf peuples de Yi 㽰, les huit de Di 狄, les sept de Rong 或 et les six de Man 彎 sont ce que l'on appelle les sihai », les «quatre ethnies des frontières », ou encore «les ethnies des quatre frontières » [Erva zhushu: vol. 24, p. 221]. 
et augmentèrent les connaissances sur les pays étrangers, les routes qui y menaient et les phénomènes naturels qui pouvaient faciliter ou entraver la navigation. L'arrivée et l'installation de marchands étrangers dans les principaux ports du pays drainèrent, pareillement, quantité d'informations sur les contrées qui jalonnaient les routes maritimes reliant la Chine à la Méditerranée ${ }^{10}$. Les connaissances en la matière ne firent qu'augmenter au cours de la dynastie Ming, époque où les problèmes de piraterie et de contrebande, ainsi que l'intensification des échanges dans les mers asiatiques, nécessitèrent une meilleure compréhension de ces espaces par les fonctionnaires chargés de ces questions et par le gouvernement qui avait à statuer à leur sujet.

Les limites de certaines mers furent encore mieux définies et de nouveaux toponymes virent le jour, mais l'effort principal de cette époque porta surtout sur un approfondissement des connaissances du milieu marin. Elle se focalisa, et pour longtemps, sur quatre expressions qui se retrouvent au centre de la représentation de l'espace océanique : hainei, haiwai, neihai 內海 (la mer intérieure) et waihai 外海 (la haute mer) ". Pour qui travaille sur les problèmes relatifs aux activités maritimes illégales le long du littoral chinois, elles recouvrent, en effet, une certaine importance, car elles délimitent souvent l'étendue des mers où se joue le sort des batailles engagées entre les forces navales et les hors-la-loi, qu'ils soient pirates, contrebandiers, ou tout simplement migrants clandestins en quête d'un meilleur sort. Est-il pour autant possible de déterminer la ligne ou bande maritime qui sépare la mer intérieure du large, voire des eaux extraterritoriales (haiwai), et de mieux appréhender ainsi la représentation des « frontières maritimes » que la classe politique chinoise s'en faisait entre le XIVe siècle et la guerre de l'Opium (1839-1842) ${ }^{12}$ ? Le repérage de cette ligne ou zone de séparation entre deux mondes marins sera-t-il pour autant exhaustif? Permettra-t-il de saisir la (ou les) signification(s) exacte(s) de ces locutions, surtout de waihai/waiyang 外洋: (la haute mer)?

10. Des documents remontant à la dynastie Song 木 (960-1279) attestent effectivement de cet élargissement de l'horizon marin : le Récit illustré d'une mission diplomatique à Korvŏ pendant l'ère vuanhe

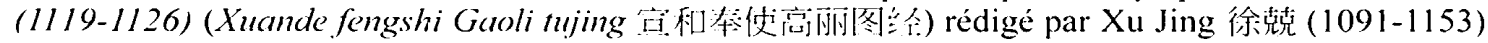
et présenté à l'empereur en 1124 (Church 2006:79-107); En guise de réponse aux questions concernant les contrées an-dela des passes (Lingwai daida 領外代管) de Zhou Qufei 周主非 (1135-1189) qui y consigna (ch. 2 et 3 ) les renseignements sur les pays étrangers recueillis auprès des navigateurs présents dans le port de Canton (traduit par Almut Netolitzky en 1977) ; la Relation sur les pays étrangers (Zhufan zhi 诸薄: à Quanzhou 宗州, qui reprend pour beaucoup les notices du texte précédent (traduction de Friedrich

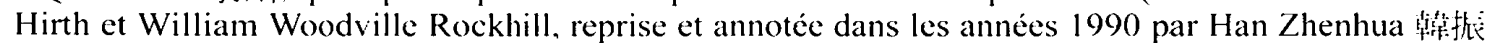
苚). Sous les Yuan 元 (1271-1368) et les Ming, d’autres ouvrages vinrent s'ajouter à cette liste, dont

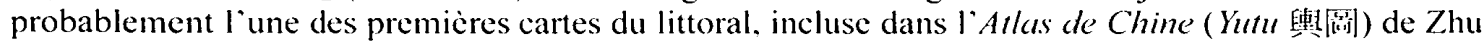
Siben 朱枈本 (1273-1337), compilé autour de 1320 (Fuchs 1946). Au sujet de ces cartes, voir Mills 1954 ; Li Xiaocong 2006 : Salmon 2006.

11. Ces quatre vocables sont éventuellement interchangeables, d'où parfois une certaine difficulté d'interprétation des sources.

12. Les travaux pionniers relatifs à la frontière maritime chinoise sont l'ouvrage de Bodo Wiethoff, China dritte Grenze. Der traditionelle chinesische Staat und der kïstennahe Seeraum (1969), suivi trois décennies plus tard par l'article de Ng Chin-keong, "Maritime Frontiers, territorial Expansion and Hai-fang" (1997). 


\section{Haifang 海防 : première référence à une politique frontalière maritime}

La première mention de ce terme se trouve dans des sources Song ${ }^{13}$, époque pendant laquelle le gouvernement participa directement aux échanges outre-mer et instaura une force navale qui devait protéger les voies navigables indispensables à sa survie ${ }^{14}$. L'expression gagna ses marques de noblesse sous les Ming. En effet, à partir de la fin du XIV $V^{e}$ siècle, la maîtrise des eaux riveraines se traduisit par l'adoption d'une politique de défense maritime (haifang) appliquée à l'ensemble des côtes. Elle avait été forgée par le fondateur de la dynastie, Zhu Yuanzhang 朱元璋 (1328-1398, r. 1368-1398), en réponse à la menace des pirates japonais (wokou 倭寇) ${ }^{15}$ et de quelques bandes rebelles disséminées le long du littoral, principalement des rescapés des flottes de Fang Guozhen 方國珍 (1319/1320-1374) et de Zhang Shicheng 張士誠 (1321-1367) ${ }^{16}$. Elle fut poursuivie à des degrés divers, surtout pour ce qui concerne l'entretien du dispositif militaire, par ses successeurs et par les premiers empereurs mandchous. L'usage du terme haifang ne commença toutefois à devenir plus systématique qu'à partir de la seconde moitié du $\mathrm{XVI}^{\mathbb{C}}$ siècle, lorsque les provinces orientales furent soumises aux attaques incessantes des wokou (1540-1560). Son emploi plus diffus reflète un changement de taille. À cette époque, la mer était en effet devenue un terrain d'affrontement entre groupes marchands rivaux et entre escadres pirates antagonistes : elle était un territoire qu'il fallait s'approprier. Cela obligea les gouvernants à repenser cet espace et surtout à délimiter et à mieux

13. Par exemple dans l'Histoire de la dynastie Song (Song shi : vol. 3, j. 47, p. 933 ; vol. 35, j. 407 , p. 12295). D'après les sources chinoises plus anciennes, des pans du littoral avaient déjà fait l'objet d'une politique défensive, attestée par l'emploi d'expressions telle que haibian wushi 活滛無雪 (le calme règne sur la frontière maritime) que l'on retrouve dès l'époque des Trois Royaumes (222-265) (San guo zhi, « Wei zhi »:j. 27, p. 4574-4b).

14. C'est à partir de cette époque que la flotte militaire fut progressivement organisée sur des bases permanentes, en particulier après la défaite de 1127 qui sonna la fin des Song du Nord (960-1127), et que ses unités furent amenées à jouer un rôle grandissant au sein de la défense territoriale. Les efforts portèrent principalement sur les voies fluviales, Huai 淮, fleuve Jaune (Huanghe 黃河) et Yangzi (Chan-

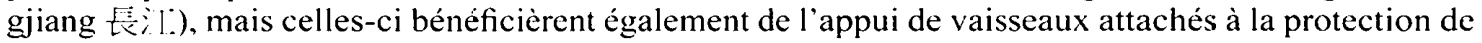
la ligne côtière et des estuaires. Avec l'arrivée de menaces étrangères, d'abord les Jin, puis les Mongols à partir des années 1230 , les unités des forces navales furent renforcées, mieux entraînées et réorganisées à plusieurs reprises. En dépit de batailles mémorables qui permirent aux Song de se maintenir encore pendant plus d'un siècle, ces efforts ne s'accompagnèrent pas des résultats escomptés. Ironie du sort, "ce fut la marine de guerre qui devait perdre les Song, après les avoir tant de fois sauvés ! ", comme l'a fort bien démontré Jacques Dars (1992: ch. 5).

15. Au XIVe siècle, les wokou étaient essentiellement des Japonais, parfois accompagnés de quelques Coréens, tandis que dans les années 1540-1560 il s'agissait de flottes aux équipages cosmopolites, Japonais, Coréens, Portugais, etc., mais comprenant une majorité de Chinois, dont étaient issus les meneurs les plus importants.

16. Fang Guozhen et Zhang Shicheng étaient deux des principaux rivaux du fondateur de la dynastie Ming. Fang Guozhen était originaire de Huangyan 黃岩 (Taizhou $\hookrightarrow 川_{1}$. Zhejiang). Aventurier des mers, il instaura un régime indépendant au Zhejiang entre 1348 et 1367 , date à laquelle il se soumit à Zhu Yuanzhang. Au cours de ces années, il avait mis sur pied une flotte importante, comptant des milliers de marins et près d'une centaine de navires. À son sujet, voir Ming shi: vol. 12,j. 123, p. 3697-3700 ; $D M B: 433-435$.

Zhang Shicheng était originaire de Baijuchang 白㓩場 (Yangzhou 揚州, Nan Zhili) et travaillait comme batelier dans l'administration des salines avant de se rebeller et de s'établir à Pingjiang 'Ý: (act. Suzhou 蘇州, Jiangsu 源蘇). Installé dans une des régions les plus riches du pays, il se proclama prince de $\mathrm{Wu}$ 吳 en 1363. À partir de 1365, Zhu Yuanzhang lança une vaste campagne contre ses territoires au nord du Yangzi dans le but de l'empêcher de s'allier aux Mongols. À la suite de cette offensive, Zhang Shicheng fut fait prisonnier et exécuté à Nankin en octobre 1367 (DMB:99-103). 
contrôler des zones stratégiques pas encore complètement intégrées au système sécuritaire de l'empire, notamment certaines îles. Néanmoins et bien que la bande côtière ait été considérée comme une frontière dès avant cette époque, l'usage du vocable haifang n'était pas fréquent, les auteurs lui préférant des expressions telles que "défense du littoral » (yanhai bianfang 沿海邊䦿). Cela s'explique peut-être par le fait que cette politique de défense ait concerné en priorité les rivages.

Il est possible, en revanche, qu'au moment de sa mise en place au début des Ming, elle ait également englobé les eaux adjacentes du littoral, car le transport maritime avait été envisagé dès la fin du $X_{I} V^{\circledR}$ siècle pour ravitailler les armées stationnées dans le nord de la Chine menacée par les Mongols ${ }^{17}$. L'établissement d'avant-postes navals sur des îles peu éloignées, au début de la dynastie, confirme d'ailleurs l'élargissement vers la haute mer du périmètre sécuritaire et la mise en place d'une politique certes modeste mais néanmoins très active. Celle-ci avait été préconisée pour contrer l'ennemi avant qu'il n'atteigne la terre ferme et prévenir l'administration et les populations de l'approche du danger. Elle reflète la pensée stratégique de l'époque : ces mesures furent en effet également employées sur la frontière septentrionale, avec l'édification d'une ligne de fortifications installée dans l'Ordos. Ce dispositif fut cependant ramené sur le continent à la fin du règne Chenghua f 成化 (1465-1487), lorsque les autorités jugèrent que ces installations étaient trop isolées en mer. Il fut de nouveau rétabli au cours des dernières années du règne Jiajing 㔛请 (1522-1566), en raison des pillages des wokou. Par la suite, sous les règnes Longqing 隆庱 (1567-1572) et Wanli 萬曆(1573-1620), puis à l'arrivée des Hollandais à partir des années 1620 , les infrastructures défensives furent encore renforcées et surtout étendues à certaines îles stratégiques, telles Nan'ao 南澳 ou celles de l'archipel des Penghu 澎湖群島 (Pescadores).

L'apparition et surtout l'emploi du terme « défense maritime » (haifang) se présente ainsi comme le reflet des tensions apparues au XIV ${ }^{\mathbb{C}}$ siècle et, plus systématiquement, à partir des années 1540 sur le front oriental et méridional du pays. À l'origine, l'application de cette politique avait d'ailleurs pris la forme d'une épreuve de force entre le gouvernement central et les habitants de ces régions, dont beaucoup furent enrôlés, déplacés, voire même déportés. Il s'agissait, de toute évidence, de mesures préventives visant avant tout le contrôle des populations côtières et navigantes. Ces méthodes draconiennes furent rarement appliquées par la suite et, lorsqu'elles le furent, elles concernèrent surtout les insulaires, les Dan 蛋 (Tanka), les trafiquants et les pêcheurs (Calanca 2011 : 257-261). L'évacuation des îles et le transfert de leurs habitants vers l'intérieur des terres fut l'une d'entre elles. Elle constitua un trait récurrent de la gestion opérée par le gouvernement chinois - tant durant les Ming que les Qing - qui espérait ainsi priver les hors-la-loi de leurs sources d'approvisionnement et tarir les échanges sur les marchés interlopes proches du continent ${ }^{18}$.

17. À ce propos, il faut signaler que sous les Yuan, en raison de l'établissement de la capitale à Dadu 大昀' (act. Pékin), le gouvernement avait déjà entrepris le transport des céréales de la région du Jiangnan vers le nord par voie maritime (Dars 1992: transport fluvial 155-157 et maritime 179-188).

18. Il s'agit d'une stratćgie que l'on retrouve aussi en France aux $x v^{\prime \prime}$ et $x v I^{\circ}$ siècles. Faute de moyens réellement engagés dans la défense du littoral. la conservation des positions avancées (îles) paraît discutable, voire illusoirc. L'évacuation des insulaires et la destruction des habitations afin de les soustraire à l'ennemi semblent alors être la meilleure solution (Bois : 2002). 
Dans l'historique de la défense maritime, le milieu du XVI ${ }^{\mathrm{e}}$ siècle constitue un point de rupture : pour la première fois, la Chine était directement menacée depuis la mer. Cette nouvelle donne rappela aux gouvernants le caractère frontalier de ces régions, déjà signalé par le fondateur des Ming, puis négligé en raison de l'absence de dangers pressants. D'importants débats s'engagèrent alors au sujet de la politique à adopter afin de sécuriser ce «nouveau » front. Son organisation militaire en fut la principale bénéficiaire : les efforts portèrent, en particulier, sur une meilleure territorialisation des unités armées qui augmenta leur opérationnalité et accrut leur rapidité d'intervention. La doctrine navale, en revanche, ne connut pas de changements significatifs et ce malgré l'intensité des discussions et des analyses. Celles-ci se focalisèrent principalement sur une seule question tactique : fallait-il choisir une politique défensive à partir d'une ligne fixe, constituée par les rivages continentaux, ou opter pour une approche plus dynamique s'appuyant sur une flotte bien aguerrie, censée intercepter l'ennemi avant qu'il ne débarque ? C'est cette seconde approche qui fut adoptée : elle s'appuya sur un triptyque composé de mesures comportant, par ordre d'importance, la " défense en mer » (vu haiyang 禦海洋), ou encore l'" arrêt de l'ennemi en pleine mer » (fangdi yu hai 防敵於海) ; la 《consolidation des lignes défensives du littoral » (gu hai an 固海岸) et un "renforcement des dispositifs de défense des villes et des ouvrages fortifiés » (yan cheng shou 嵌城守) ${ }^{19}$. Si, dans la théorie, la priorité fut ainsi donnée à une stratégie défensive active, son application s'avéra problématique.

Zheng Ruozeng 鄭若㴊 (1505-1580) ${ }^{20}$, en bon chroniqueur de cette période, exposa, dès le début du chapitre consacré aux stratégies de gestion de cette crise, l'un des problèmes qui empêchait la mise en pratique de ces mesures : " les fonctionnaires civils ne prennent pas la mer, tandis que les commandants militaires fuient les risques et n'osent s'aventurer sur l'eau ${ }^{21}$. Jusqu'au $x^{2}$ siècle et à l'exception de quelques rares périodes,

19. Sur ce sujet, voir l'ouvrage de Zheng Ruozeng, Chouhai tubian. L'auteur y a consigné pour ainsi dire l'ensemble des renseignements relatifs aux événements, aux débats, aux politiques choisies qui ont marqué les années 1540-1560. En ce qui concerne les stratégies envisagées à cette époque, se reporter en particulier au chapitre 12 .

20. Zheng Ruozeng était natif de Kunshan 它山 (Suzhou, Nan Zhili) ct son pc̀re, contrairement à la tradition familiale tournée vers les lettres, s'était engagé dans les activités commerciales. Il travailla de longues années comme conseiller militaire dans l'état-major de Hu Zongxian 胡扩隹 (151 1-1565). Hu Zongxian était originaire de Jixi 縤溪 (Huizhou 徽州, Nan Zhili) et obtint son titre de docteur en 1538. À partir de 1554, il participa activement à la lutte contre les wokou au Zhejiang, puis fut responsable de la défense côtière l'année suivante. En 1556, il fut nommé commandant suprême du Nan Zhili, du Zhejiang et du Fujian, et occupa ce poste pendant plus de six ans. En 1560, il fut, avec d'autres, accusé de détournement de fonds, puis à nouveau en 1565 , ce qui lui valut d'être emprisonné pour corruption (Ming shi : vol. 18,j. 205, p. 5410-5415).

21. Chouhai tubian :j. 12, p. 764. Zheng Ruozeng rapporte ici les observations de Tang Shunzhi 唐順: (1507-1560). Des propos qui en rappellent d'autres, même s'ils ne relèvent pas du même registre, tenus par Lan Dingyuan 藍寎元 (1680-1733) en 1717, lorsqu’il dénonçait l'interdiction faite aux Chinois de se rendre dans le Nanyang, et qui lui faisait dire que cette décision avait été prise par des ministres à la science livresque, dépourvus de toutes connaissance du littoral, tandis que ceux qui en disposaient n’étaient pas écoutés à la cour (Luzhou chuji,j. 3, p. lb, plus généralement sur ce sujet, p. la-6a).

Tang Shunzhi était natif de Wujin 此進 (Changzhou 潮州, Nan Zhili) et obtint le titre de docteur en 1529. Essayiste et critique littéraire de renom, il mena de pair une excellente carrière officielle qui le vit, à la fin de sa vie, participer à la lutte contre les wokou (DMB: 1252-1256).

Lan Dingyuan était originaire de Zhangpu 浐浦 (Zhangzhou) et ne réussit jamais à passer les examens officiels, ce qui ne l'empêcha pas d’accéder au poste de magistrat de district (xianzhi 絲知1) et à celui de préfet de Canton juste avant sa mort. Il travailla comme secrétaire et conseiller personnel auprès 
la gouvernance de ces régions dut s'accommoder de cette difficulté. Comme le faisait remarquer Tan Lun 譚綸 (1520-1577) ${ }^{22}$, un des plus brillants stratèges de l'époque : "Ceux qui discutent aujourd'hui des affaires maritimes ont constamment à la bouche la 'défense de la ligne côtière', [tandis qu'] il vaudrait mieux affronter la menace en mer. En réalité, la mer est un espace sans limites. Alors, par où faut-il [commencer] la défense? Depuis que les calamités se sont abattues [sur le littoral], jamais une seule de nos unités n'a réussi à anéantir [l'ennemi] en mer, pas plus qu'à le contraindre de s'en retourner d'où il était venu. Quand bien même il nous arrive d'entraver leur marche, une à deux fois sur dix, ces incidents ne sont nullement susceptibles d'entamer ses ambitions, ils sont juste semblables aux coups de vent. Par contre, sur terre, il est possible de l'anéantir complètement et de l'empêcher de revenir piller nos territoires. L'impact des combats terrestres et navals est bien différent ! " ${ }^{23}$. Ainsi, à défaut de disposer d'une marine opérationnelle, il convenait indirectement que les batailles continentales étaient à privilégier. Parmi les représentants du gouvernement, la faction qui prônait un renforcement du système défensif de la façade côtière l'emporta et la question relative à la constitution d'une flotte aguerrie, tranchée après la guerre de l'Opium, n'eut de répercussions significatives qu'au $\mathrm{XX}^{\mathfrak{E}}$ siècle, semble-t-il, plus par manque de financement que de volonté d'innovation. Toutefois, et il s'agit là de l'une des plus grandes contributions du milieu du Xví siècle aux questions maritimes. C'est à partir de ce moment que le littoral cntra concrètement et définitivement dans le débat sur la sécurité de l'empire.

Jusqu'à cette époque, la frontière maritime avait, en effet, été la grande absente des traités de défense territoriale qui ne prenaient en considération que les confins septentrionaux, comme le montre très clairement le Catalogue des ouvrages relatifs aux frontières chinoises (1958) de Deng Yanlin 悔衍林 (1908-1980). De nombreux ouvrages traitant spécifiquement de la défense maritime, résultats de ces débats, furent édités à la suite de la crise des années 1540-1560 et jusqu'aux premières décennies du XVII siècle ${ }^{24}$. Les premières cartes spécifiques à la côte furent également dressées à cette époque, dont celle intitulée Dix mille li de défense maritime (Wanli haifang tu 萬星海防圆), compilée par Zheng Ruozeng qui rencontra un vif succès dès sa publication et pendant des décennies. Il en fut de même pour le Précis de défense maritime ${ }^{25}$, du même auteur, édité en 1562 sous le patronage de Hu Zongxian, alors commandant suprême (zongdu 總督) du Nan

de certains hauts fonctionnaires nommés sur la côte. En 1721, lors de la révolte de Zhu Yigui 朱 出殅 (1721) à Taiwan, il participa, aux côtés de son cousin Lan Tingzhen 藮起珍 (1664-1730), alors général de la brigade de Nan'ao, à la campagne de répression, dont il relata les faits dans son Ping Tai ji $\gamma^{3}$ st (Notes sur la pacification de Taiwan), ouvrage d'importance pour les observations qu'il y consigna sur l'administration de l’île, son exploitation agricole et son système de défense contre une éventuelle attaque de l'extérieur.

22. Tan Lun était originaire de Tanfang 1544. Sa biographic est souvent associéc à celle de Ji Qiguang avec qui il collabora étroitement, aussi bien dans les provinces sur-orientales que plus tard sur la frontière septentrionale.

23. Chouhai mbian: j. 12, p. 770-771.

24. Certains d'entre eux connurent d'ailleurs un grand succès éditorial et leurs nombreuses éditions successives apparurent le plus souvent lorsque le littoral connaissait des périodes de crise.

25. Les sept premiers chapitres de cet ouvrage forment une sorte de géographie stratégique de la côte, circonscription par circonscription. 
Zhili 南直隸 ${ }^{26}$, du Zhejiang 浙汭. et du Fujian 福建. Sous la dynastie Qing, les atlas du littoral (yanhai 叱海), de la «défense maritime" (haifang) ou encore de la " frontière maritime " (haijiang) sont plus nombreux, signe de l'évolution de la situation dans cette partie du pays et surtout de sa prise en compte par le gouvernement et les officiers chargés d'y faire régner la paix. Les cartes les plus intéressantes, ou du moins celles qui semblent avoir bénéficié d'une certaine notoriété, furent en effet souvent dressées par des fonctionnaires militaires, parfois civils, en poste dans ces régions.

\section{Haijiang 海疆 : apparition de la frontière maritime}

L'intrusion de l'espace littoral dans le discours sécuritaire à partir du milieu du XVI" siècle ne modifia pas tout de suite le lexique en usage. Jusqu'aux années 1650 , il n'était pas encore question de la « frontière maritime » (haijiang); cette expression apparut pour la première fois dans cette acception sous le règne Shunzhi 順治 (1644-1662) de la dynastie Qing (1644-1911), mais ne fut employée de façon plus systématique qu'avec l'irruption des puissances européennes ${ }^{27}$. Jusque-là, cette notion était exprimée par d'autres locutions, faisant surtout référence à la protection des rivages marins. De même que la notion de défense maritime, l'expression «frontière maritime » fut, malgré son usage peu fréquent, le reflet des événements survenus à cette époque, au cours de laquelle les combats faisaient rage entre les Mandchous qui tentaient d'asseoir leur pouvoir au sud de la Chine, principalement au Fujian, et la famille Zheng qui luttait pour maintenir ses privilèges, sa mainmise sur le commerce transocéanique et son indépendance. Son usage dans les années 1650 s'insère dans le contexte de crise qui déchira la Chine sud-orientale : 1656 sonna le glas des pourparlers entre le gouvernement mandchou et Zheng Chenggong et marqua le début de l'« expédition du Nord » entreprise par ce dernier qui le mena, de conquête en conquête, jusqu'aux portes de Nankin (1656-1659). La transition dynastique Ming-Qing - marquée par la dissidence des Zheng, la révolte des Trois gouverneurs militaires (San fan zhi luan 三藩之亂, 1674-1681) et la guerre que leur opposa le nouveau régime - a constitué, de même que les ravages des wokou au milieu du XvI" siècle, une nouvelle étape dans le long processus d'intégration du littoral au discours sécuritaire du gouvernement. Au terme de cette période, au début des années 1680 , les provinces côtières ne pouvaient plus être considérées comme une zone périphérique que le gouvernement pouvait se permettre d'ignorer ( $\mathrm{Ng}$ Chin-keong $1997: 212$ ).

C'est pendant cette période que les populations littorales subirent une des politiques les plus draconiennes que la région ait jamais connue : l'évacuation de la côte (qianjie 遷界

26. Le Zhili méridional incluait une partie du Jiangsu et de l'Anhui actuels, ainsi que la région de Shanghai. Le terme zhili signifie " directement administré » par le gouvernement central. Cette juridiction fut créée sous la dynastie Ming et incluait les circonscriptions autour de la capitale. L'adjectif “méridional » lui fut ajouté pour le distinguer du Zhili septentrional (Bei Zhili 化直隸), lorsque la capitale fut transférée à Pékin en 1421, sous le règne Yongle 永樂 (1403-1425). À l'avènement des Qing, elle fut ramenée au statut de province et porta le nom de Jiangnan 江南.

27. La première mention que nous avons trouvée de ce terme date du 20 janvier 1656 (Qing shilu, Shizu Zhang Huangdi shilu : vol. 3, p. 753). Il apparaît également dans l'Histoire de la dynastie Ming (Ming shi : vol. 4,j. 40, p. 881 ; vol. 22,j. 259, p. 6720 ; vol. 27,j. 322), ouvrage rédigé entre les dernic̀res décennies du XvII et les premières du XvIII' siècle. Dans les autres sources, en particulier les histoires non officielles et les écrits personnels, il est surtout employé à partir de la seconde moitié des Qing, à

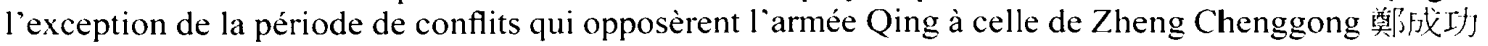
(1624-1662, Koxinga dans les sources européennes). 
ou encore qianbian 遷邊), c'est-à-dire l'application d'une stratégie de la « terre brûlée 》 ou, pour utiliser une expression chinoise, de «nettoyage des campagnes » (qingye 清野). Cela impliqua le départ forcé des populations, la destruction de leurs habitations, de leurs navires et même de certaines fortifications édifiées ou restaurées un siècle auparavant, lors des attaques des wokou. Au Fujian, dans le cadre de ces dispositions, une muraille, en partie financée par les habitants, fut même érigée le long de la façade maritime. Il s'agissait d'un rempart flanqué de fortins et de points d'appui placés à distance fixe, très variable en termes de taille et matériaux : par endroits, c'était un mur haut de six pieds (environ $1,90 \mathrm{~m})$ et large de quatre $(1,28 \mathrm{~m})$, tandis qu'ailleurs il n'était constitué que d'une simple haie de branchages. Par cette mesure, le gouvernement espérait isoler le rebelle (Zheng Chenggong) et empêcher tout soutien continental. Le premier édit à ce sujet date du 14 octobre 1660 et ne concerna que deux localités proches de Xiamen 廈門: Paitou 排頭, dans le district de Tong'an 同安 (Quanzhou), et Fangtian 方用, dans celui de Haicheng 海澄 (Zhangzhou 漳州) ${ }^{28}$. L'année suivante, les populations du Jiangnan, du Zhejiang, du Fujian et du Guangdong 廣東 durent à leur tour quitter les rivages et s'installer dans l'arrière-pays. Ce sont les habitants de ces deux dernières provinces qui eurent le plus à en souffrir. Au Zhejiang, seules les préfectures de Ningbo 寧波, Taizhou et Wenzhou 溫州 furent concernées, tandis qu'au Jiangnan, la cour suivit l'avis du général de division, Liang Huafeng 梁化鳳 (?-1671), qui se prononça contre l'application de cettc disposition. Seules les préfectures de Suzhou 蘇州 et de Songjiang 松江 furent affectées, car elles étaient dans le périmètre d'activité choisi par Zheng Chenggong jusqu'à la fin des années 1650 (Zhejiang tongzhi $1899: j$. 71, p. 1b; Qing shi gao: vol. 32,j. 243, p. 9596).

Il s'agit là de mesures militaires qui s'inscrivent dans le contexte conflictuel des années 1640-1680. Les empereurs mandchous montrèrent, en effet, plus d'intérêt pour le commerce maritime que leurs prédécesseurs et, sauf à deux reprises, ils n'empêchèrent pas les Chinois d'en faire. Dans le discours officiel, le négoce outre-mer est perçu comme un facteur de stabilité sociale. Dans le domaine de la protection des rivages, en revanche, les Qing n'apportèrent pas de modifications structurelles à la politique Ming, si ce n'est en institutionnalisant le processus amorcé au sein de l'organisation de l'armée à la suite de la crise des wokou, que le gouvernement précédent n'avait pas eu le temps de pérenniser: l'extension du "système de défense frontalier » à l'ensemble des provinces ${ }^{29}$. Concrètement, cela se traduisit par un meilleur rayonnement des implantations militaires, en particulier sur les principales îles longeant la ligne côtière, où les contingents disposèrent désormais d'une assise permanente et non plus rotative comme par le passé. Cette évolution marque-t-elle pour autant un changement de stratégie? Les longues tergiversations qui ponctuèrent le processus d'incorporation de Taiwan confirment, comme cela avait été le cas pour l'archipel des Penghu et Nan'ao, la résistance que soulevait encore, au sein du gouvernement, l'extension du dispositif sécuritaire par le stationnement d'effectifs stables loin du continent. Elles soulignent, en revanche, l'inéluctabilité de ces avancées au vu non pas d'une évolution de la réflexion stratégique, mais tout simplement du développement économico-politique de ces régions depuis la fin du XvI ${ }^{\circledR}$ siècle.

28. Qing shilu, Shizu Zhang Huangdi shilu: vol. 3,j. 140, p. 1081 ( 11 ‘ jour du $9 *$ mois de la $17^{\circledR}$ année du règne Shunzhi).

29. Sur l'évolution du système militaire, voir Calanca $2011: 163-255$. 
Les fonctionnaires et les officiers qui eurent à se pencher sur la question de la défense maritime au début des Qing, lorsqu'il fallut réorganiser économiquement et militairement le littoral sud-oriental, avaient parfaitement intégré les leçons des années 1540-1560 ${ }^{30}$. En effet, si l'incorporation des îles les plus éloignées (Taiwan) rencontrait encore des résistances, celles qui balisent les eaux côtières furent, pour les principales, toutes investies par des contingents militaires, d'importance variable suivant l'intérêt stratégique de leur position. La mise en place de ce cordon extracontinental fut le résultat de l'expérience et de la dynamique qui caractérisa le début de la dynastie Qing, une fois la paix revenue. Les fonctionnaires qui la préconisèrent s'inscrivent en effet dans l'héritage des penseurs et des hommes d'action qui furent au premier rang de la lutte contre les wokou, à commencer par Zheng Ruozeng pour qui l'examen de la situation géostratégique était essentiel. Ils disposèrent ensuite de l'écoute attentive de l'empereur, sans qui rien n'aurait pu être envisagé. Il s'agit là de la seule avancée dont bénéficia ce dispositif défensif jusqu'aux Guerres de l'Opium (1839-1842 et 1856-1860) ${ }^{31}$.

Une fois les unités militaires installées aux points stratégiques, la politique de sécurité des rivages s'appuya, comme précédemment, sur une ligne défensive avant tout continentale, soutenue par une législation visant à contrôler l'accès des habitants à la mer (haijin). Sur cet aspect, le gouvernement mandchou procéda à un important effort de réglementation et infléchit sa politique vers une plus ample ouverture du commerce. Celle-ci conserva néanmoins un caractère restrictif, en particulier au regard des garanties sociales et financières nécessaires à l'obtention de la licence d'armer des navires pour l'étranger. Bien que ces autorisations ne fussent pas facilement accordées, les habitants vécurent, à partir de cet instant, dans un contexte de limitation de ces activités et non plus de prohibition, comme cela avait été le cas sous la dynastie Ming. L'interdiction de commercer vers l'étranger ne fut en effet adoptée que lors du conflit qui opposa le gouvernement aux Zheng et, à nouveau, non sans de nombreuses oppositions, entre 1717 et 1727 pour celui à destination de l'Asie du Sud-Est ${ }^{32}$. Les temps de la prohibition étaient, à cette époque, quasiment révolus, et cette politique restrictive n'apparaissait plus comme une solution que pour une frange minoritaire de fonctionnaires. Ceux-ci profitaient, en ce début de $\mathrm{XVII} \mathrm{I}^{\circ}$ siècle, du fragile équilibre atteint par les provinces méridionales et de la méfiance de l'empereur Shengzu 聖祖 (r. 1662-1722) envers ces populations pour agiter le spectre de la contrebande des grains et des migrations illégales vers l'outre-mer.

30. C'est au cours de la dernière décennie du XVII" siècle que quelques-uns des ouvrages les plus significatifs parus à la suite de la " période wokou » furent réédités, dont le Précis de défense maritime.

31. Pour un aperçu concis au sujet de la pensée et du travail de ces hommes, se reporter à l'ouvrage de Wang Hongbin (2002, partic 4).

32. Il semblerait que la prohibition n`ait pas concerné toutes les provinces, comme le laisse entendre la biographie de Gao Qizhuo 其倬 (1676-1738), gouverneur général du Min-Zhe de 1725 à 1727 . puis au même poste au Fujian jusqu'en 1730, lorsque cette charge fut instaurée au Zhejiang et au Fujian (Qing shi liezhuan : vol. 4,j. 14, p. 1062. Ce passage se trouve également dans Qing shi gao : vol. 34, j. 292, p. 10303).

Gao Qizhuo était membre de la bannière chinoise jaune à bordure et obtint son titre de docteur en 1694 . À son sujet: Qing shi gao : vol. 34,j. 292,p. 10301-10305; Qing shi liezhuan : vol. 4,j. 14, p. 10581064 : $E C C P: 721,735,920$. 


\section{Bornage des sphères maritimes d'intervention}

C'est également sous la dynastie Ming que les vocables neihai et haiwai, ou neiyang 內숭 et waiyang, commencèrent vraisemblablement à recouvrir une nouvelle sphère d'action du gouvernement. La mer étant devenue un espace convoité, il fallait pouvoir en assurer la sécurité en s'appuyant sur un dispositif défensif approprié, dont les unités étaient désormais astreintes à œuvrer à l'intérieur d'un territoire élargi. Le terme waihai fut employé dès la dynastie Song, mais il désignait, à cette époque, avant tout les pays étrangers. Les recherches menées dans les sources Ming n'ont apporté aucune définition précise de ces expressions, mais attestent de leur usage, principalement en relation avec le service de garde-côtes, la pêche et la piraterie. Il s'agit le plus souvent de témoignages de poursuites ou de victoires remportées contre les pirates en haute mer ${ }^{33}$. Les pages réservées aux mesures mises en place dans le périmètre d'intervention de la garnison de Changguo 珀國衛 et dans le district de Dinghai 定海 au moment de la crise des wokou révèlent que des efforts pour localiser certaines îles avaient été faits, mais ces précisions sont encore très peu nombreuses (Chouhai tubian : j. 5). Bien qu'aucun examen exhaustif à ce sujet n'ait été réalisé, il semble que l'usage de l'expression « en pleine mer » (zai haizhong 在海中), aux contours plus vagues, soit encore très fréquent. Elle est d'ailleurs encore régulièrement employée dans les mémoires des fonctionnaires en charge du littoral à l'époque mandchoue.

La démarcation entre ces espaces - mer intérieure, haute mer, mer extérieure - relève, chez les navigants, du visuel comme du physique : la haute mer se situe là où la côte n'est plus perceptible à la vue ; son «bornage » passe là où la topographie et les phénomènes naturels (hydrographie et météorologie), en conditionnant la navigation et la perception des eaux, jouent l'un des rôles principaux dans sa définition. Sa délimitation prend appui suivant le découpage de la ligne côtière, la morphologie des fonds marins ou encore le cycle des marées, comme le suggère un texte du début du XVII siècle, bien que la proposition ne soit pas très probante : " la surface de la mer est immense et ne peut être mesurée par aucun procédé ; seul le cycle de la marée [permet] d'en distinguer [ses espaces] $\gg{ }^{34}$. Il pourrait ainsi être question de la ligne de base servant de nos jours encore à fixer la limite de la mer territoriale, correspondant à la laisse de basse mer en deçà de laquelle se situent les eaux intérieures ${ }^{35}$. Le contenu d'un mémoire au trône de la même époque, relatif à l'organisation de la surveillance des voies navigables du Jiangnan, principalement le Yangzi, fournit d'autres éléments et délimite assez précisément cette frontière, du moins pour les embouchures des grands fleuves : " la haute mer (dayang 大洋) est très éloignée de l'arrière-pays ; [l'espace] qui la borde s'appelle 'estuaire' (haikou 海 $\square$ ). Les navires souhaitant pénétrer à l'intérieur des terres s'engagent tous par là pour accéder au port. Des batteries y ont été érigées. Les estuaires les plus vastes dépassent les cent $l i$, [depuis leur bordure jusqu'aux] villages [continentaux], les plus étroits plusieurs

33. À ce sujet, voir par exemple, (houhai tubian :j. 4-6, 8-10, 12.

34. Zhejiang tongzhi, Shanghai, Shanghai guji chubanshe : vol. 1,j. 3, p. 257.

35. Depuis 1982, la mer territoriale est calculée à 12 milles marins (soit 22,224 km) à partir de la laisse de basse mer, c'est-à-dire la limite de la zone de l'estran qui n'est que rarement découverte par les eaux. 
dizaines. À l'intérieur de ceux-ci, la partie la plus étendue est nommée 'mer intérieure' (neiyang), la plus petite est communément appelée 'rade/baie' (haitao 海素) $)^{36}$.

Pour ce qui concerne la limite entre mer intérieure et haute mer, loin des embouchures stratégiques, les renseignements sont rares et moins précis. Le texte le plus détaillé semble ĉtre cclui de la Monographie générale du Zhejiang, déjà cité par Li Xiaocong, où il est question des limites territoriales (jiangyu 疆域) du district de Dinghai. Les zones sont circonscrites suivant les quatre directions cardinales à partir de lieux précis : « À l'est, [dans la région comprise] entre Shenjiamen 沈家門 et la pointe Tangtou 塘頭踭, les localités de Putuo 普陀, de la Grande et Petite Luojia 大小洛伽, de Zhujiajian 朱家尖, de Shuci 樹梑, de Yangyu 洋嶼, de Liangheng 梁橫, de Hulu 葫眬 et de Baisha 6 沙 [...] se situent toutes dans la mer intérieure. À l'est de Langgang 浪岡, celle de Fushan 福II; au nord de la Grande et Petite Qushan 大小眐II.., celles de Xiangpeng 鯐蓬, de Zhaizi 寨子 et Donggualan 東瓜爛 [...] sont toutes localisées en haute mer ». Certains passages semblent encore plus explicites sur la segmentation de cette zone autour de Dinghai : «Au sud, la mer intérieure arrive jusqu'à Liuheng 六横 qui délimite la frontière avec le district de Zhenhai 鎮海縣, [tandis] qu'à l'ouest c'est Jintang 金塘 qui marque les confins territoriaux avec l'île de Jiaomen 蛟門山, [toujours] dans le district de Zhenhai ; au nord, on pénètre dans la haute mer à partir des îles de Yangshan 洋ll et Xugong 徐公山 qui départagent les eaux du Jiangsu 江蘇 et du Zhejiang; [...]» ${ }^{37}$ (voir carte p. 54).

Les espaces marins ainsi désignés et bornés furent progressivement circonscrits entre la fin du XVII et le début du XVIII ${ }^{\mathrm{e}}$ siècle, sous l'impulsion des empereurs Shengzu et Shizong 世宗 (r. 1723-1735) ${ }^{38}$. Cette terminologie est-elle pour autant explicite ? Cet effort de démarcation a-t-il touché l'ensemble du littoral? La rareté des renseignements recueillis, leur formulation et leur emploi n'ont pas encore dissipé la totalité des incertitudes qui les entourent. Ils suscitent surtout de nouvelles interrogations : comment en effet ne pas remarquer que les rares passages qui traitent explicitement de la délimitation entre mer intérieure et eaux hauturières concernent, après les estuaires, les îles ? Dans le cas de Dinghai cité ci-dessus, parmi les territoires insulaires, ceux qui se situent à l'intérieur des eaux côtières forment une sorte de couronne autour de Zhoushan, tandis que ceux qui s'érigent au large constituent visiblement une barrière protectrice, aux abords de l'estuaire du fleuve Qiantang 錢塘江. Ces eaux, intimement liées au continent, étaient fréquentées depuis toujours et constituaient le «terroir » traditionnel de vie et de travail des habitants des provinces sud-orientales. Leurs contours devaient probablement appa-

36. Il s'agit d'un mémoire au trône daté du 28 novembre 1728 (27 jour du $10^{\mathrm{C}}$ mois de la $6^{\mathrm{e}}$ année

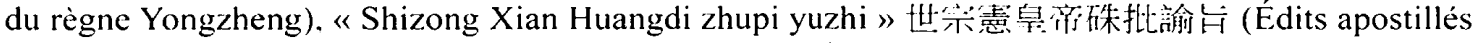
par l'empereur Shizong, Xian huangdi de son nom posthume), in Wenyuan ge Siku quanshu 1983 : vol. 421,j. 126-14, p. 421-412; cité par Li Xiaocong 2006:164. La signification donnée dans ce texte au mot « rade » correspond également à l'une des définitions de ce terme en français, c'est-à-dire le plan d'eau qui sépare parfois de la pleine mer les ports ou les baies

37. Zhejiang tongzhi : vol. 1,j. 3, p. 257-258.

38. De nouvelles cartes pourraient réapparaître, dans le sillage de la Carte de la défense maritime du

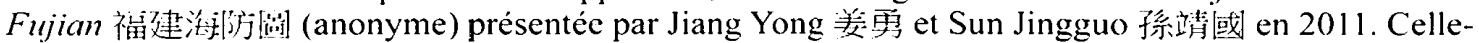
ci remonterait aux années 1597-1604 et semble très précise, notifiant l'emplacement des ouvrages défensifs, les distances d’un lieu à un autre, les vents, les courants, les conditions de mouillage, etc. Elle est conservée à la bibliothèque de l'Académie des sciences. Il semble évident que, depuis le milieu du $\mathrm{XVI}$ " siècle, la nouvelle réorganisation des forces navales établie pour mettre fin aux ravages des wokou se soit également accompagnée d'un effort cartographique qui devait soutenir le travail des officiers en poste sur le littoral. 
raître moins nets à ceux qui n'en avaient pas une expérience directe, principalement les fonctionnaires civils. Pour ces derniers, la mer était, le plus souvent, sans limites (wuya 無涯), d'où les difficultés pour l'appréhender, la gérer et la sécuriser, d'où probablement aussi une certaine timidité dans la gestion de cette frontière mouvante.

\section{La pratique de la mer entre « neihai » et « haiwai »}

Le service de garde-côtes fut certainement une des missions les plus importantes et les plus difficiles qui furent assignées aux unités militaires stationnées le long des rivages marins. Il comprenait de nombreuses tâches : la surveillance et la protection des ports, des feux de vigie, des postes de contrôle sur le continent et sur les îles adjacentes ; l'escorte des bâtiments de transport officiels ; la protection des navires de commerce et de pêche ; des opérations de secours en mer, des naufrages aux simples avaries nécessitant un remorquage, etc. Organisé en fonction des saisons, il comportait l'inspection des routes maritimes, la vérification des navires et de leurs équipages, et la poursuite des embarcations suspectes. Il constituait, pour finir, un exercice d'amarinage pour les troupes et leurs commandants. Les équipes se relayaient à tour de rôle et chacune des patrouilles devait faire la jonction avec les autres, de façon à couvrir l'ensemble des eaux proches du continent. Les mémoires au trône rédigés à ce sujet laissent entrevoir l'intense activité qui régnait en mer, où pêcheurs et marchands ne cessaient de se croiser le long des côtes et de se rassembler en toute saison, laissant finalement peu de répit aux hommes chargés de la surveillance de cette frontière. Qu'en était-il en réalité ?

Si les recherches dévoilent l'avance de la Chine en matière de codification navale, militaire et civile, et l'effort entrepris dès le début des Ming afin d'assurer la sécurité des rivages par l'établissement d'escadres régionales, certains témoignages suggèrent que cette organisation souffrit rapidement de nombreux dysfonctionnements, imputables aussi bien aux hommes d'armes stationnés en province et aux autorités locales qu'au gouvernement. Les avancées du dispositif militaire mis en place à la fin du XIV ${ }^{\mathrm{e}}$ siècle, renouvelé après les ravages des wokou au milieu du $X V l^{\mathfrak{e}}$ siècle, n'avaient, tout d'abord, pas résisté aux contraintes financières de la fin des Ming, comme le rapporte Chen Renxi 陳仁錫 (1579-1634) ${ }^{39}$. Ainsi, dans les années 1620-1630, alors que les pirates hantaient de nouveau la zone de cabotage, le quota des navires et les effectifs détachés dans les avant-postes navals avaient diminué des deux tiers et certaines îles étaient seulement fréquentées par les hors-la-loi, sans que l'armée disposât des moyens de les investir (Chen Renxi : j. 75, p. 1986-1987). Ȧ cette époque, le gouvernement n'était déjà plus en mesure d'assurer la défense du littoral sud-est, la menace des Mandchous accaparant toute son attention et ses efforts. D'où certainement l'« abandon », dans les années 1630, de cette tâche à Zheng Zhilong 鄭说龍 (1604-1661), pirate et aventurier originaire de Nan'an 南安 (Quanzhou), dont la flotte était conséquente et les équipages bien entraînés ${ }^{40}$.

Au début des Qing, après le retour de la paix, les souverains s'étaient, à plusieurs reprises, inquiétés de la situation en mer, consternés par l'impuissance des unités mili-

39. Chen Renxi était originaire de Changzhou tit (Sushou, Nan Zhili) et obtint son titre de docteur en 1622. Il compila un important Recueil institutionnel (Huang Ming shifa lu), d'où sont extraits ces commentaires.

40. À ce sujet, voir par exemple Blussé 1990 . 
taires et des autorités locales, incapables de réprimer les pirates et d'enrayer l'émigration clandestine et la contrebande. Des mesures avaient été prises afin de s'assurer le concours de bons officiers, en facilitant par exemple le recrutement de commandants du $\mathrm{cru}^{41}$, et de constituer un parc de navires performants. Néanmoins, à la fin des années 1730, l'état des forces navales nouvellement réorganisées était déclinant. À cette époque, par exemple, elles avaient déjà perdu 20 à $30 \%$ de leurs embarcations pour cause de détournement d'une partie des fonds alloués à leur entretien ou de réaffectation des bâtiments qui allaient grossir les rangs de la marine marchande ${ }^{42}$. La rigueur budgétaire et les économies imposées par le gouvernement contribuèrent aussi à l'affaiblissement de la flotte de guerre qui vit dès lors se creuser l'écart de compétitivité entre ses vaisseaux et les navires civils. Les différents articles réglementant la gestion des escadres militaires laissent en effet transparaître le souci financier qu'elle impliqua et, de toute évidence, les sommes qui lui furent destinées ne correspondaient pas aux besoins ${ }^{43}$. À ces difficultés structurelles vint se greffer un autre problème, cause d'autres dysfonctionnements : la connaissance, voire la méconnaissance, du terrain dont disposaient les troupes aussi bien que le gouvernement. Les unités militaires cantonnaient effectivement le plus souvent leurs opérations aux limites de leur juridiction, voire même parfois ne prenaient pas la mer. Que ce soit par crainte de se laisser dessaisir des mérites d'une opération menée loin de leur lieu de rattachement par des collègues peu prévenants, par simple méconnaissance du terrain, ou encore faute d'équipement adéquat, la lutte contre la piraterie et la contrebande eut beaucoup à souffrir de ces défaillances. Des fonctionnaires n'avaient pas hésité à les signaler, ni à rappeler la nécessité de poursuivre les bandits au-delà du périmètre assigné, mais ces recommandations semblent avoir rarement été appliquées. Des exercices de surveillance conjoints avec des bataillons navals de différentes unités stationnées le long du littoral avaient été instaurés en $1716^{44}$. Cette mesure fut-elle suivie d'effets?

Rien n'est moins sûr, car Gao Qizhuo 崮其倬 (1676-1738) en était encore à la recommander en 1725, dès son arrivée au Fujian en qualité de gouverneur général du Min-Zhe 閩浙 (Fujian-Zhejiang). Dans son souci d'améliorer les connaissances nautiques des troupes, il suggéra alors qu'une fois par an et suivant le mouvement saisonnier des vents le service de garde-côtes soit dirigé par des officiers aptes à naviguer jusque dans les eaux des provinces limitrophes, leur tournée devant également inclure les îles situées au large du continent ${ }^{45}$. Cette suggestion fut formulée à la suite d'une inspection des hommes d'armes qui révéla que les tours de surveillance étaient limités aux eaux toutes

41. Sur le recrutement des officiers de la marine, voir Calanca 2007.

42. Les officiers auraient en effet rédigé de faux rapports, en notifiant la réparation des navires quand en réalité elle n'était effectuée que sur quelques-uns, les autres étant tout simplement repeints afin de leur donner belle allure. Les fonctionnaires auraient également pris une part active au commerce avec le Japon et l'Annam en y envoyant des membres de leur famille ou de leur parenté, ou encore des marchands à qui ils allouaient, en contrepartie de bénéfices, des embarcations de l'armée [Qing shi gao : vol. 14.j. 135. p. 3984 ; (Qinding) Da Qing huidian shili $1908: j .936$. p. 2b. L'ouvrage sera désormais abrégé en $D Q H D S L$, éd. Guangxu].

43. DQHDSL. éd. Guangxu:j. 936, p. la-4a.

44. DQHDSL. éd. Guangxu :j. 632, p. $1 \mathrm{~b}$.

45. Qing shi gao : vol. 14,j. 135, p. 3982. 
proches et qu'une forte naupathie affectait les soldats et les officiers ${ }^{46}$. La proposition fut acceptée par le gouvernement car, dès 1725, les navires du Zhejiang et du Fujian furent astreints, en dehors de leur service de reconnaissance régulier, à participer une fois l'an à un entraînement commun en haute mer. Réitérée à plusieurs reprises dès avant le milieu du XVIII" siècle par des commandants qui défendaient son utilité, il n'est pas sûr que cette pratique ait connu une application sur le long terme ${ }^{47}$. Certains officiers ne cachaient d'ailleurs pas ces défaillances, comme en 1807, lors d'une opération de répression contre le pirate Zhu Fen 米濆 (?-1809). Ce dernier étant entré dans les eaux du Guangdong, les officiers de l'escadre du Fujian furent contraints d'interrompre leur traque aux environs de Nan'ao, en raison de l'infériorité numérique de leurs troupes et de leur manque d'expérience des hauts fonds ${ }^{48}$.

Dans de telles circonstances, les pirates disposèrent souvent d'une grande liberté de mouvement, en raison justement de leur accoutumance à l'élément marin, comme le soulignait déjà en 1678 le gouverneur général du Fujian, Yao Qisheng 姚啟聖 (1624-1684) :

Les pirates du Fujian +"), depuis [l'avènement] de l'actuelle dynastie, n'ont cessé pendant des décennies de venir semer le trouble, coûtant des sommes considérables au gouvernement, faisant du tort à d'innombrables soldats mandchous et chinois. fauchant une multitude de vies parmi la population. Les raisons de leur force résident entièrement [dans leur vie] à bord des navires : la mer est leur foyer, le bateau toute leur vie. Leur équipement (voiles, mâtures, cordages, canons, etc.) est de première qualité. En fonction de la direction et de la couleur des nuages, ils prévoient la venue des vents particuliers. Ils connaissent tous les secrets des airs et ne se trompent jamais là-dessus. Ceci n'est pas le propre des pirates, mais de tous ceux qui vivent dans ce milieu, y passent toute leur existence et $y$ célèbrent les naissances, les décès comme les mariages. Du haut commandant aux petits soldats, tous vivent à bord des navires, c'est pourquoi ils y sont bien accoutumés et sont valeureux au combat.

Actuellement, si notre armée aperçoit les pirates attaquer les frontières, cavaliers et fantassins se hâtent à leur poursuite, mais ne peuvent chaque jour parcourir que quarante

46. Gongzhong dang Yongzheng chao zouzhe : vol. 6, p. 823-827 : mémoire daté du 01/12/1726 ( $8^{\circ}$ jour du $11^{\circ}$ mois de la $4^{\circ}$ année du règne Yongzheng).

47. Voir, par exemple, Archives historiques n"1 (Pékin). Gongzhong zhupi zouzhe, junwu, 11 : mémoire

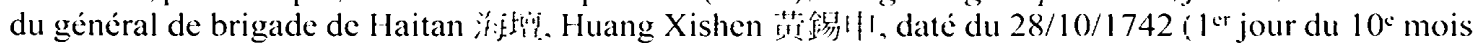
de la 7 année du règne Qianlong).

Huang Xishen était originaire de Pinghe $\psi$ 秛l (Zhangzhou).

48. Archives historiques n' I (Pékin): Gongzhong zhupi zouŁhe. junw'u-fangwu. 43 : mémoire du

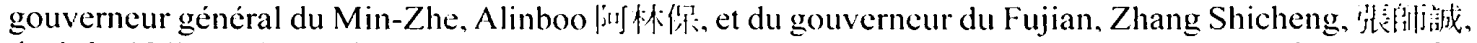
daté du 12/05/1807 (5' jour du $4^{*}$ mois de la $12^{\circ}$ année du règne Jiaqing): Gongzhong zhupi zouzhe. nongmin yundong, 75, 1076/2: mémoire du gouverneur général du Liang-Guang. Wu Xiongguang 拉熊光 daté du 10/05/1806 (22 jour du $3^{\circ}$ mois de la $11^{\circ}$ année du règne Jiaqing).

Alinboo était membre de la bannière blanche unie.

Zhang Shicheng (1762-1830) était originaire de Gui an 鼠父: (Huzhou 湖州, 7hejiang) et obtint son titre de docteur en 1790. Il fut gouverneur du Fujian de 1806 à 1814 . période pendant laquelle les escadres de pirates, bien que constituant toujours une menace pour la sécurité des navigants, avaient été affaiblies par l'action des troupes gouvernementales. Il assista notamment à la mort de Cai Qian 誃参: (?-1809). tué lors d'un combat naval (Qing shi g(to : vol. 37,j.359. p. 11360-11362:ECCP:499,511).

Wu Xiongguang (?-1833), originaire de Zhaowen $\|_{1}^{3 j}$ 文 (Suzhou. Jiangsu), était détenteur du titre de licencié.

49. L'auteur fait ici principalement rérérence aux troupes de Zheng Chenggong. 
ou cinquante $l i$, tandis que, tout à leur aise dans leurs embarcations, les pirates surgissent en un instant. Les efforts fournis par les deux armées ne sont pas comparables. $[\ldots]^{50}$

Quelques années plus tard, au début du XvII ${ }^{\mathrm{e}}$ siècle, un autre témoin, Xu Xudan 徐旭旦 (?-1714), exprimait encore des réflexions désabusées sur l'état de la défense et la nécessité de disposer d'équipages amarinés :

L'usage des bateaux par les forces navales est semblable à celui que font les troupes terrestres des chevaux. Si ces derniers ne galopent pas toute la journée, hommes et bêtes s'accoutumant les uns aux autres, lorsqu'ils se rangeront en ordre de bataille, ils craindront à tout instant de tomber. Comment pourraient-ils tuer les bandits? Si les navires de l'armée ne naviguent pas sans relâche, les hommes et les embarcations travaillant en harmonie, lorsqu'ils se déploieront pour la bataille ils redouteront sans cesse de se renverser. Comment pourraient-ils aspirer à la victoire ? À présent, les vaisseaux de guerre mouillent au port et de toute l'année on ne les a pas vus bouger. Si le matériel n'est pas mancuvré, il risque fort de pourrir et de finir en morceaux au bout de quelques années (Qing jingshi wenbian : vol. 3,j. 83, p. 2046).

En 1800, l'empereur faisait remarquer que les soldats et petits gradés des postes de contrôle des préfectures du littoral n'attachaient aucune importance à l'inspection en mer (DQHDSL éd. Guangxu :j. 630, p. 5a).

Cette timidité face à l'élément marin, voire même simple tactique pour se préserver des périls physiques (blessures, noyades, etc.) et des sanctions disciplinaires ${ }^{51}$, ne caractérisa

50. Kangxi tongyi Taiwan dang 'an shiliao xuanji : 164-166. Par la suite, d'autres fonctionnaires eurent l'occasion de signaler l'habileté des pirates, sur mer comme au combat: Archives historiques n" 1 (Pékin), Gongzhong zhupi zouzhe, “Nongmin yundong»㟽民連動 (Mouvements paysans), 1046/2, 74 : mémoire conjoint du gouverneur général du Min-Zhe, Yude F德, et du gouverneur du Fujian, Li Diantu 专殿圖, daté du $21 / 08 / 1804$ ( $17^{\circ}$ jour du $7{ }^{\circ}$ mois de la $9^{\circ}$ année du règne Jiaqing).

Yude (?-1809) était membre du clan Gūwalgiya 厎网伴 et de la bannière jaune unie. Il fut nommé sur la côte sud-est en 1796 en qualité de gouverneur du Zhejiang, puis promu au poste de gouverneur général du Min-Zhe (1800-1806). Dans la lutte contre la piraterie, il entretint des relations très difficiles avec Li Changgeng 李是庈 (1750-1808), dont l'action fut souvent entravée par ses interventions. Il fut finalement démis de ses fonctions et banni en raison de son incapacité à combattre les pirates.

Li Diantu (1738-1812) était originaire de Gaoyang 高陽 (Baoding 保定, Bei Zhili) et obtint son titre de docteur en 1766. Il fut gouverneur du Fujian de 1802 à 1806. Il avait déjà travaillé dans cette province en qualité de juge, de 1795 à 1798 , ce qui lui permit de se familiariser avec les coutumes locales et de régler quelques cas de rixes entre groupes coalisés. Il fut néanmoins destitué de sa fonction de gouverneur pour ses faiblesses en matière militaire, mais reconnu par ses successeurs comme un bon administrateur (Qing shi gao: vol. 37,j. 359, p. $11359-11360$; ECCP : 349).

Li Changgeng était originaire de Tong'an, tout comme son plus farouche ennemi, le pirate Cai Qian. Il est surtout connu pour les mesures qu'il entreprit pour combattre la piraterie, soutenues par le gouverneur du Zhejiang, Ruan Yuan l沅汒 (1764-1849, éminent fonctionnairc et homme de lettres), puis entravées par Yude. Il décéda lors d'un combat naval avec la flotte de Cai Qian.

51. Les règlements relatifs à la remise en état ou au remplacement des bâtiments de la flotte ayant subi des avaries au cours d'opérations en mer ou lors de typhons prévoyaient en effet des sanctions pécuniaires et disciplinaires pour les militaires qui en avaient la charge. Ainsi, si des détériorations se produisaient lors d"un accrochage avec les pirates, les officiers pouvaient être démis de leur fonction et obligés à rembourser le navire. Dès les premières années du règne Shunzhi (1644-1662), des peines furent adoptées à l'encontre de ccux dont les navires avaient été endommagés au cours du service de gardecôte : pour deux embarcations détruites, le responsable perdait deux degrés, mais restait en fonction : de trois à quatre, il subissait la même peine, mais il était muté ; de cinq à six, quatre degrés lui étaient 
pas uniquement les troupes locales lors de leur travail de surveillance ; on la retrouve également au sein même de la stratégie maritime du gouvernement. Elle influença en particulier le processus d'incorporation des îles au continent, ainsi que leur administration après leur intégration à l'empire. Les propos ci-dessus cités de Yao Qisheng et Xu Xudan suggèrent clairement une distinction entre le périmètre arpenté par les riverains de la mer et celui sillonné par les représentants de l'État. Le premier répondait aux nécessités de la survie, en relation avec un milieu écologique précis; le second se limitait aux zones stratégiques proches du continent et était ainsi plus réduit. Les bordures de l'espace fréquenté s'arrêtaient, pour les premiers, aux limites de leurs connaissances et de leurs compétences en matière de navigation, ainsi que de la catégorie et de la capacité nautique de leurs embarcations, et incluaient, du lieu le plus proche au plus lointain, les baies, les estuaires, ainsi que les îles et îlots adjacents jusqu'à ceux qui se situaient en haute mer, certains desquels relevant de la mer extérieure ${ }^{52}$. Pour les seconds, elles étaient le plus souvent confinées à la sphère d'action du service des patrouilles, soit une ligne statique qui pouvait, suivant les époques et les commandants, inclure ou exclure jusqu'aux terres émergées proches des rivages.

Le champ d'intervention des forces navales semble avoir été très mouvant, se réduisant et s'élargissant en fonction de l'habilité et des aptitudes manifestées par les officiers ou de la conjoncture en mer, ce qui ne facilita pas le travail d'appréciation des événements par le gouvernement à Pékin. En 1703, l'empereur Shengzu, craignant que l'armée ne fasse preuve de trop de timidité dans la lutte contre la piraterie, ordonna de dresser des cartes, afin de pouvoir juger de la valeur des informations qui lui parvenaient et être en mesure de suivre les agissements des pirates et les poursuites menées par l'armée ${ }^{53}$. Les officiers invoquaient en effet souvent, comme le rapporte quelques années plus tard l'ancien gouverneur général du Min-Zhe, Fan Shichong 範恃崇 (?-1721), le franchissement par les bandits des limites relevant de leur compétence territoriale, pour justifier de leurs maigres résultats ${ }^{54}$. Il devenait alors indispensable, avant même de s'interroger

enlevés et il était transféré ; au-dessus de sept, il était remercié. La rétrogradation, même accompagnée de l'autorisation de conserver son poste, équivalait à une diminution du traitement, et il n'est donc pas étonnant que les fautifs aient fréquemment tenté de cacher les accidents. La loi était, en revanche, plus clémente lorsqu'il s'agissait de dégâts imputés au mauvais temps. Les autorités supérieures étaient alors chargées d'enquêter et lorsque l'investigation innocentait les militaires de toute responsabilité, ils étaient dispensés de payer (DQHDSL, éd. Guangxu :j. 631, p. 6a;j.632, p. 1b).

52. Dès le $x v^{e}$ siècle, les pêcheurs de la côte orientale se rendaient dans les îles japonaises les plus

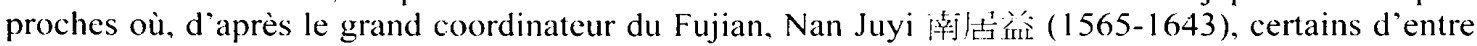
eux se seraient installés et auraient fondé des foyers avec les femmes de la région (Ouyang Zongshu 1998: 178-179).

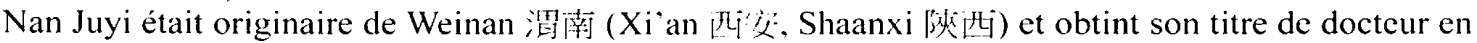
1601. Il est entre autres connu pour avoir réussi à déloger les Hollandais des îles Penghu et les habitants du Fujian, pour le remercier d’avoir éloigné ce danger, érigèrent des temples en son honneur ( $D M B$ : 1085-1088).

53. Qing shilu, Shengzu Rén Huangdi shilu : vol. 6,j. 213, p. 161: entrée datée du 25/10/1703 ( $15^{\circ}$ jour du $9^{\circ}$ mois de la $42^{\circ}$ année du règne Kangxi).

54. Qing shilu. Shengzu Ren Huangdi shilu: vol. 6,j. 268, p. 627 : entrée datée du 5/04/1716 ( $12^{\circ}$ jour du $3^{\circ}$ mois de la $55^{\circ}$ année du règne Kangxi).

Fan Shichong était originaire de Shenyang 沋陽 et appartenait à une famille affiliée à la bannière jaune

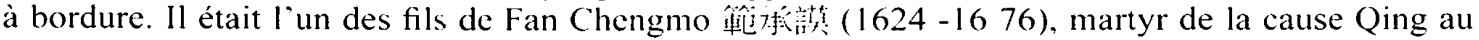
moment de la rébellion des Trois Gouverneurs militaires (San Fan) (ECCP : 228-229; Qing shi gao : vol. 32,j. 252, p. 9723-9725; Qing shi liezhuan : vol. 2,j. 6, p. 380-383). 
sur la pertinence ou non d'étendre les chasses en mer loin des rivages ${ }^{55}$ - un des thèmes les plus récurrents dans les réflexions relatives à la défense côtière-, de pouvoir appréhender le territoire entre la mer intérieure (neihai ou neivang) et la haute mer (waihai ou waiyang), voire l'Outre-mer ou la «mer extérieure » (haiwai). Fan Shichong avait alors proposć d'imposer aux plus hautes instances provinciales la tenue de registres répertoriant les noms des différents emplacements et leur localisation.

Les difficultés d'accès aux cartes du littoral et à leurs catalogues dans certaines bibliothèques ne permettent pas de savoir si celles dont l'établissement fut exigé par l'empereur Shengzu en 1703 existent encore de nos jours. Celles-ci ont pu être réalisées sous différents formats, ce qui rend encore plus difficile leur localisation : rouleaux, feuilles cartonnées, atlas, ou encore feuillets annexés aux mémoires rédigés à l'adresse de la cour. Quant aux registres de Fan Shichong, aucun d'entre eux n'a été retrouvé pour l'instant, mais les monographies générales du Jiangnan et surtout du Zhejiang, dans leur édition du règne Yongzheng (1723-1736), laissent comprendre que ces délimitations de l'espace maritime ont été effectuées par écrit et vraisemblablement graphiquement aussi. Celle du Jiangnan montre que ce souci était déjà présent dans les années 1680, au moment où la piraterie recommença à faire parler d'elle. Les chapitres consacrés à la défense côtière, à l'armée (bingzhi 兵志) ou encore aux préparatifs militaires (wubei zhi 武備 论), y compris ceux de la monographie du Fujian, révèlent que des postes de contrôle, parfois désignés comme «navals 》 (shuixun 水汛), furent installés dans des lieux situés en pleine mer. Les soldats y étaient vraisemblablement détachés sur une base fixe, tandis que leurs officiers - souvent un seul par poste - l'étaient alternativement, par périodes de deux mois ${ }^{56}$. Au fur et à mesure de l'évolution des activités économiques et malgré la volonté du gouvernement, le dispositif sécuritaire s'étendit progressivement aux îles proches. Il s'agit certes d'une lente et timide avancée vers le large, mais elle apparaît de façon inéluctable dès le milieu du $\mathrm{XVI}^{\mathbb{C}}$ siècle. Il reste encore à comprendre les raisons qui incitèrent les auteurs des cartes à représenter Taiwan, les Penghu et Hainan hors de

Fan Shichong occupa, avant d’être nommé gouverneur général du Min-Zhe (1710-1715), le poste de gouverneur du Guangdong (1705-1710), où il eut l'occasion de se prononcer en faveur du repeuplement des îles évacuées en 1662. Lorsqu'il arriva au Fujian, les autorités étaient aux prises avec les pirates, dont Zheng Jinxin 鄭洪心 qui fut capturé l’année suivante (Qing shi liezhuan : vol. 3,j. 12, p. 849-852; Qing shi gao : vol. 32,j. 252, p. 9725).

55. La correspondance administrative consigne plusieurs cas de ce type, ainsi que la connivence entre pirates et bandits de l'intérieur : Kangxi chao hanwen zhupi zouzhe huibian : vol. 4, p. 511-516: mémoire du général provincial du Zhejiang, Wu Jun 点郡 (Wu Jun [?-1715], originaire de Pucheng 浦城 - Jianning 建管, Fujian), daté du 27/11/1712 (29 jour du $10^{\circ}$ mois de la $\left.5\right|^{\circ}$ année du règne Kangxi) ; vol. 4, p. 664-668 : mémoire du général de Canton, Guan Yuanzhong 管源思 (un Chinois des bannières), daté du 13/02/1713 (19 jour du $1^{\mathrm{er}}$ mois de la $52^{\circ}$ année du règne Kangxi) ; vol. 5 , p. 230-232 : mémoire du général provincial du Guangdong, Wang Wenxiong E文倠 (?-1723, originaire de Daxing 人與. Shuntian 順天), daté du 03/12/1713 (16 jour du $10^{\circ}$ mois de la $52^{\circ}$ année du règne Kangxi) ; Archives historiques no 1 (Pékin), Gongzhong zhupi zouzhe 宮中硃批楿搝 (Mémoires au palais apostillés), junwu 算冴 - fangwu 防枒 (Affaires militaires, défense frontalière), 10 : mémoire

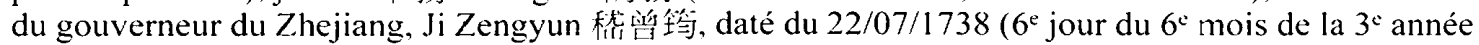
du règne Qianlong).

Ji Zengyun (16 71-1739) était originaire de Changzhou l三 1706. Spécialiste des questions hydrauliques, il fit une partie de sa carrière à des postes à haute responsabilité dans ce domaine. Il est l'un des auteurs de la Monographié générale du Zhejiang déjà citée.

56. Zhejiang tongzhi : vol. 2,j. 94, p. 1703. 
leur contexte géographique. En effet, elles apparaissent souvent à la fin du rouleau ou de l'ouvrage cartographique.

\section{L'intégration des îles : une lente avancée vers le large}

Pendant la période considérée, Hainan 海南 était la seule île d'une certaine importance à jouir d'un long rattachement administratif à l'empire, même si elle était encore, au début des Qing, considérée par certains continentaux avec appréhension, tout juste bonne à servir de lieu d'exil pour les criminels et les opposants (Xu Chonghao $1975: j .5$ ). Néanmoins, et en dépit d'un contrôle plutôt lâche du gouvernement, le peuplement han était désormais bien enraciné sur son pourtour, même si les relations qu'entretenaient ces communautés avec les populations autochtones, principalement les Li, demeuraient difficiles. Un autre trait différenciait Hainan des autres territoires insulaires : elle ne semble pas avoir fait office de terre d'asile pour les hors-la-loi et les contrebandiers, cela s'expliquant peut-être en partie par la présence d'une population établie depuis longtemps, organisée et donc policée, ainsi que par l'implantation relativement ancienne des agences étatiques ${ }^{57}$. Ce qui n'était pas encore le cas, au xvII' siècle, pour Zhoushan et l'archipel portant le même nom, situés au sud de la baie de Hangzhou 杭州, constituant la plus importante pêcherie de Chine et détenant une position hautement stratégique pour la sécurité de l'empire.

Cette région avait connu un essor sous la dynastie Song ${ }^{58}$, mais par la suite, son intégration au continent avait présenté un processus beaucoup moins linéaire que celui de Hainan. Au cours des deux dernières dynasties, elle s'était à plusieurs reprises trouvée au centre de graves contentieux : au début des Ming, les pirates japonais et des bandits chinois rôdaient dans ses parages ; au milieu du XVI" siècle, l'île de Shuangyu 雙典, en particulier, devint un important centre interlope, rassemblant des groupements cosmopolites d'aventuriers de tous bords ; durant la transition dynastique, Zhoushan fut à plusieurs reprises disputée par les belligérants et pour finir, du moins pour la fin de la période qui nous concerne, elle fut enlevée par les Anglais en 1840. D'après les témoignages relatifs à ce dernier événement, il semblerait que les troupes chinoises, prises au dépourvu, furent très rapidement mises en déroute (Waley 1959: 108-109; Ward Fay $1975: 219-225$ ). Au-delà de l'aspect inattendu de cette attaque, il est probable que le dispositif sécuritaire souffrait, comme dans d'autres localités côtières, de dysfonctionnements variés, dont le premier était une présence étatique insuffisante, principalement en soldats et vedettes assurant la sécurité en mer, comme le soulignait déjà en 1742 le général de la brigade

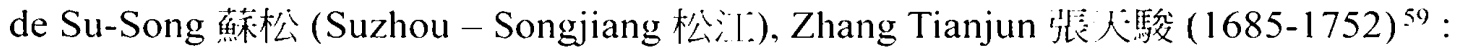

57. Au sujet de la piraterie à Hainan, voir Calanca 2001 : 111-136. Sur l’île en général, voir Ptak \& Salmon 2001.

58. Au sujet de l’historique de la présence étatique à Zhoushan, voir Moll-Murata 2006: 109-123.

59. Zhang Tianjun était natif de Renhe 1 : 利: (Hangzhou), suite à un changement de lieu de résidence de la famille, originellement installéc dans la région de Wenzhou. Il fut promu dans les forces navales du Fujian après s'être distingué en tant que lieutenant à la capitale. Ayant remporté des victoires sur les pirates, il gravit les échelons de la hiérarchie jusqu’au poste de général provincial du Guangdong.

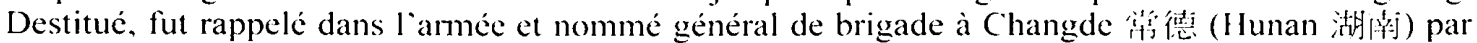
décret spécial (tezhi 特花), puis muté à Chongming 
À chaque saison de pêche. il est fréquent que des malfaiteurs pillent et causent des dommages aux marchands et aux pêcheurs. Votre serviteur, lorsqu'il s'est enquis de la situation, [s'est aperçu que] les grands navires de surveillance n'arrivent pas à imposer leur autorité (1).

D'après les mémoires relatifs au service de garde-côtes, la saison de la pêche était effectivement, pour les autorités, une période particulièrement éreintante, vécue avec hantise. Les sources laissent transparaître la crainte des officiers d'être dẻbordés par deux ou trois milliers de navires, dont la présence impliquait une surveillance et une protection redoublées. La concentration de bateaux en un lieu précis et à une époque donnée rendait très difficile le travail des patrouilles qui avaient du mal à identifier les mâts et devaient manœuvrer entre les filets en tâchant de ne pas perturber l'activité, se retrouvant souvent dans l'impossibilité de poursuivre les embarcations suspectes ${ }^{61}$. Même en temps de paix, les autorités locales avaient le sentiment de ne pas disposer du nombre d'hommes et du matériel nécessaires à leur tâche.

À l'exclusion de quelques îles (Hainan, Xiamen, Jinmen 全門, etc.), la grande majorité des territoires insulaires n'était pas vraiment contrôlée par le gouvernement et bien des années après leur rattachement et après avoir été investis par des contingents militaires, certains d'entre eux étaient encore perçus comme des lieux peu recommandables. Situés en pleine mer, ils pouvaient constituer, dans l'imagerie bureaucratique, le havre idéal pour les repris de justice, principalement les pirates qui parfois y élisaient domicile ${ }^{62}$. La description de l'archipel des Penghu donnée par les auteurs de l'Histoire de la dynastie Ming laisse percevoir un sentiment d'impuissance :

Ces éminences éloignées se dressent au milieu de la mer comme de longs serpents sinueux. Les ports naturels abondent dans ces îlots ; certains sont si vastes qu'ils peuvent servir de cachette à de grands navires ${ }^{6.3}$.

un homme intègre et loyal, il semble avoir connu un certain succès auprès de ses hommes [(Chongriu) Taiwan fuzhi : vol. 2,j. 11, p. 1997-1998].

60. Zhang Tianjun ajoute qu'il avait d’ailleurs chargé les pêcheurs honnêtes de surveiller les événements

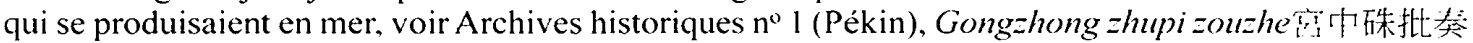

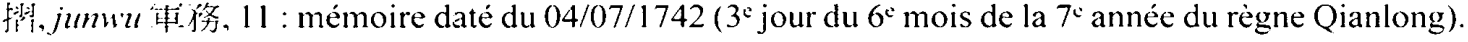

61. Archives historiques n"1 (Pékin), Gongzhong zhupi zouzhe,junwu-fangwu軍狢一防枒, 10-11:

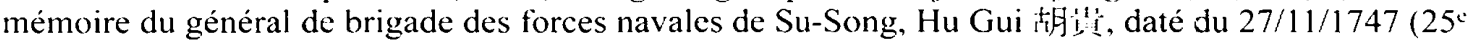
jour du $10^{\circ}$ mois de la $12^{\circ}$ année du règne Qianlong). Ces remarques s`appliquent aussi à la région de Sansha “沙 (Funing 福密i, Fujian), en particulier en hiver lorsque les navires des préfectures méridionales affluaient pour la saison de la pêche. Les officiers avaient alors des difficultés à distinguer les bons éléments des mauvais [Gongzhong dang Yongzheng chao zouzhe : vol. 4, p. 65-67 : mémoire du général de brigade de Funing, Yan Guangwu 顏光旿, daté du 01/05/1725 (19e jour du $3^{\circ}$ mois de la $3^{*}$ année du règne Yongzheng)].

Hu Gui (?-1760) était originaire de Tong`an et poursuivit sa carrière dans la marine militaire jusqu’au poste de général de division du Guangdong.

Yan Guangwu (?-1729) était originaire de Bozhou 㲤州, Jiangnan. Au cours de sa carrière, il se serait distingué par son attitude. Au Fujian, des témoignages épigraphiques expliquent qu`il aurait pris soin de ses troupes et, selon la formule consacrée (xingli chubi 與利除桨婪), participé à promouvoir le bien public et à supprimer les abus. Il serait mort de maladie à son poste.

62. À ce sujet, voir par exemple la description de Zhoushan dans le chapitre géographique de la monographie qui lui est relative (Zhoushan zhi:21).

63. Ming shi : vol. $8, j .91$, p. 2247. 
Au début du XVIII" siècle, une grande ignorance semble encore caractériser leur situation au regard de certains propos tenus par l'empereur Shengzu excluant la possibilité qu'elles eussent pu servir de repaires aux pirates ${ }^{64}$. Cette hypothèse avait été corroborée par les autorités à la suite de témoignages de repentis qui les décrivaient comme des lieux inhospitaliers en raison de la pauvreté des sols et de l'insalubrité de l'eau. Il est néanmoins difficile d'accréditer cette thèse car, contrairement à ces affirmations, bon nombre d'entre elles étaient habitables, sans pour autant être très accueillantes. D'après le témoignage de Chen Minglong 陳明隆, un ancien acolyte du pirate Zheng Jinxin 鄭盡心 ${ }^{65}$, capturé au mois de janvier 1711, Jinshan(hua) 盡山(花) (dans l'archipel de Shengsi 嵊泪, à l'extrême sud du Jiangsu), Yushan 泌山l (autre archipel relevant de la préfecture de Taizhou au Zhejiang) et les îlots au large de Danshui 淡水 (Taiwan), constituaient les bases à partir desquelles Zheng Jinxin et sa bande organisaient leurs expéditions ${ }^{66}$. Leur position, située sur le passage des navires chargés de grains destinés à la capitale et aux provinces déficitaires en riz, était hautement stratégique pour le contrôle de l'ensemble du trafic maritime, de cabotage et de haute mer (voir carte p. 54).

Souvent présentées comme étant " isolées dans la mer extérieure » (guxuan haiwai 孤䋰海外), elles ont tardé à jouer un rôle décisif au sein du dispositif sécuritaire. Proches ou éloignées, l'administration y avait établi des agences suivant une logique et des impératifs complexes, pas nécessairement liés à la distance qui les séparait du continent, ni à leur position stratégique. Ce processus intervint le plus souvent après des périodes de crise, lorsque le gouvernement s'était vu contraint d'y établir des bureaux ou de les investir avec des unités militaires afin d'éviter qu'elles ne deviennent des repaires de hors-la-loi ou des bases stables pour des régimes étrangers. Il en avait été ainsi aux Penghu, à la suite de l'arrivée et de l'installation des Hollandais au début des années 1620. L'annexion de Taiwan à l'empire (1684) constitue certainement un des meilleurs exemples à ce sujet. La décision de lancer une expédition contre l'île, afin de venir à bout du régime dissident que la famille Zheng y avait installé, ne fut prise qu'après des années de tergiversations. Au-delà des aléas d'une bataille navale, loin de tout appui continental, du risque d'insurrection populaire au Fujian ${ }^{67}$ et de l'éternelle querelle entre partisans de la répression et de la pacification, le principe de l'expédition soulevait également un

64. Kangxi (has hanwen zhupi zouzhe huibian : vol. 4, p. 313 : mémoire du gouverneur du Zhejiang, Wang Duzhao 1: I t: $H_{1}^{\prime}$, daté de juillet-août 1712 (6 mois de la $51^{\circ}$ année du règne Kangxi).

Wang Duzhao était originaire de Zhucheng 渚城 (Qingzhou docteur en 1685 . Il a été gouverneur du Zhejiang de 1710 à 1714.

65. Zheng Jinxin était originaire de Fuzhou 襍州 et fut capturé autour du 24 avril 1711 , en compagnie d’une cinquantaine de complices. D’abord condamnés à mort, ils furent graciés en raison de leur bonne connaissance de la mer et du maniement des armes, puis envoyés au Heilongjiang comme matelots [Qing shilu, Shengzu Rén Huangdi shilu: vol. 6,j. 245, p. 435 : entrée datée du $24 /(04 / 1711$ (7c jour du $3^{\circ}$ mois de la 50" année du règne Kangxi)].

66. Kangxi chao hanwen zhupi zouzhe huibian : vol. 3, p. 221 (220-223) : mémoire du gouverneur

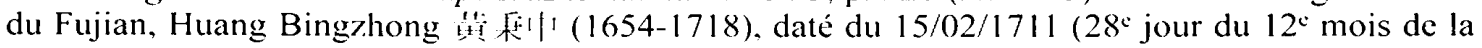
$49^{\circ}$ année du règne Kangxi).

Huang Bingzhong, membre de la bannière chinoise rouge à bordure, était originaire de Haicheng

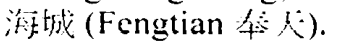

67. Le sud du Fujian, berceau de la famille Zheng, devint, de ce fait, le principal théâtre des combats avec toutes les conséquences funestes que près de quarante ans de guerres ont infligé à la population et à l'économie locales. 
problème important, où l'élément psychologique n'était pas exclu, dans la mesure où les opérations devaient avoir lieu au-delà de la "mer intérieure » (neihai), voire même de la « haute mer» (waihai), en dehors du périmètre couvert normalement par le dispositif défensif du littoral. Il s'agit ici bien entendu de la vision des hommes politiques, car les pêcheurs et les marchands fréquentaient depuis longtcmps un espace bien plus vaste qui comprenait certaines localités de Taiwan. Entre la fin du XVI ${ }^{\circ}$ et le début du XVII siècle, des escadres de la flotte militaire y avaient d'ailleurs déjà patrouillé afin d'empêcher les pirates et les Japonais de s'y installer. Ces escadres étaient dirigées par des commandants courageux et surtout expérimentés, dont la renommée s'est perpétuée bien après leur disparition, tel Shen Yourong 沈有容 $(1557-1627)^{68}$.

Les empereurs mandchous, tout comme leurs prédécesseurs, n'étaient pas prêts à élargir le domaine maritime sous leur juridiction. À l'origine, l'expédition contre Taiwan ne fut envisagée que comme une opération de police, discutable parce que située à l'extérieur de l'empire, mais nécessaire pour réduire les rebelles qui menaçaient les rivages. Pour une partie du gouvernement, la question devait se clore avec le rapatriement des habitants après la victoire des troupes impériales. Shi Lang 施琅 $(1621-1696)^{69}$, convaincu depuis toujours de l'importance stratégique de sa position et de ses potentialités économiques, réussit finalement, aidé en cela par d'autres fonctionnaires travaillant au Fujian, à convaincre le conseil impérial du bien-fondé de cette annexion. Bien avant l'archipel des Penghu et Taiwan, Nan'ao avait connu un sort identique, car ce ne fut qu'en 1575, à la suite des assauts répétés du pirate Lin Feng 林鴯 ou Lin Afeng 林阿鳰 (Limahong dans les sources européennes), qu'un contingent y fut installé et dont le rayon d'action s'étendait des eaux relevant du Fujian méridional à celles placées sous juridiction du Guangdong oriental $^{70}$. À noter que Liu Yaohui 劉椝誨 (1522-1585), grand coordinateur du Fujian de 1573 à 1576, avait été l'un des promoteurs de l'extension du dispositif sécuritaire à cette île : un officier qui s'était distingué pour sa vision offensive de la défense côtière, n'hésitant pas à lancer ses troupes au-delà du périmètre assigné, voire même outre-mer (dans le cas présent, les Philippines), pour poursuivre les hors-la-loi ${ }^{71}$.

Ces trois exemples démontrent que ces lieux n'ont véritablement attiré l'attention du gouvernement qu'après être devenus des zones interlopes, obligeant les autorités à prendre en considération leur position stratégique et l'enjeu qu'ils représentaient pour

68. Shen Yourong était originaire de Xuancheng 宣城 (Ningguo 综淢, Anhui) et décida d'embrasser la carrière militaire bien qu'il descendit d'une famille de lettrés. En 1579, il passa avec succès l'examen militaire provincial et se distingua au cours de plusieurs batailles. Il travailla, en particulier, au Fujian dès 1597 où il occupa la charge de commandant d'avant-poste (bazong 把總) à Wuyu 法嶼, puis à Shihu 不湖. Sa carrière s'est presque entièrement déroulée dans les provinces du bord de mer (Ming shi : vol. 23,j. 270, p. 6938-6939; DMB : 1192-1195; sa biographie se trouve dans de nombreuses monographies locales du Fujian).

69. Shi Lang, originaire de Jinjiang (Quanzhou), était issu de l'une des grandes familles du littoral. Il travailla d'abord sous les ordres de Zheng Zhilong et cessa de servir les Zheng lorsque Zheng Chenggong tua une partie de sa famille. Il se soumit alors aux autorités Qing et participa à leurs côtés à de nombreuses campagnes au Fujian. Il fut l'artisan de la conquête de Taiwan.

70. Nan' 'so zhi, éd. Qianlong :j. 3, p. 4b.

71. Liu Yaohui était originaire de Linwu 倏留武 (Hengzhou 衡州, Huguang 湖廣) et obtint son titre de docteur en 1553. À son sujet : Ming shi : vol. 18,j. 205, p. $5413 ;$ vol. 19,j. 221, 5815: vol. 27,j. 31 , p. $8215: D M B: 919.929$ et 1133 . 
la sécurité du littoral ${ }^{72}$. À la fin du $x v l^{c}$ siècle, des fonctionnaires comme Xu Fuyuan 徐浮遠 (1592-1594) avaient suggéré le repeuplement de certaines îles précisément afin de les intégrer au dispositif défensif ${ }^{73}$. Grand coordinateur du Fujian, il rétablit les activités agricoles sur Haitan 海壇 (act. Pingtan 谭, Fuzhou), Nanri 南目 (Xinghua 興華) et les Pescadores, et sollicita l'autorisation de les développer aussi au Zhejiang, à Chenqian 陳錢 (autre nom de Jinshanhua), Jintang (Ningbo), Putuo shan (archipel de Zhoushan), Yuhuan 玉環 (sous les Ming préfecture de Taizhou, puis à partir de 1728 elle est rattachée à la préfecture de Wenzhou) et Nanji 南皆 (préfecture de Wenzhou) (Ming shi : vol. 7,j. 77, p. 1886). Dans sa stratégie, l'occupation et l'essor économique de ces lieux situés en mer devaient contribuer à éviter qu'ils ne deviennent des havres pour horsla-loi et endiguer l'avancée des pirates. Argument auquel le gouvernement, plus enclin à abandonner ces terres émergées au milieu de la mer, fut peu sensible.

Dès les premières années de la dynastie des Ming, la cour eut en effet recours à l'évacuation des populations et à leur installation sur le continent pour combattre l'insécurité côtière. Bien que l'édification de points d'appui fortifiés sur ces territoires désormais déserts ait été préconisée en contrepartie par certains, ils furent souvent voués à l'abandon pur et simple ${ }^{74}$. Au début du règne Hongwu (1368-1398), ces exodes forcés furent, en particulier, organisés au Zhejiang, au Fujian el dans l'est du Guangdong. Dans la première de ces provinces, ils avaient surtout concerné les îles de Jintang et de Daxie 大榭, dans la préfecture de Ningbo ; de Yuhuan et Gaopi 高不, dépendant de Taizhou; de Nanji et Dongluo 東洛, sous la juridiction de Wenzhou. Il s'agissait de terres fertiles que les habitants durent abandonner sous peine de mort (Chen Renxi 1965:j. 75, p. 1995). Au Fujian, le sort réservé aux insulaires ne fut pas meilleur : seuls les habitants de Xiamen et Wuyu, dans la préfecture de Quanzhou, furent épargnés en raison de la présence des bataillons de Zhongzuo 位: (Xiamen) et de Jinmen (Zhu Weigan 1986: 175). Ces évacuations constituèrent un véritable gâchis au regard du développement économique atteint par certaines des zones concernées. Dans le district de Funing 福崽, Fuying 浮腐 et Yushan '俞l/ étaient densément peuplées et dotées de plusieurs mouillages, et pour la seconde d'un sol fertile ${ }^{75}$. Dans celui de Fuqing, les pertes semblent avoir été encore plus importantes. Haitan, la plus grande île fukiénaise, dont la population comptait six à sept cents « groupes de responsabilité fiscale» $(l i \text { 里 })^{76}$, avait été « abandonnée aux

72. Sur l’histoire du peuplement des îles par les habitants de la côte, voir Li Deyuan 2006.

73. Xu Fuyuan était originaire de Deqing 徳消 (Huzhou 湖州, Zhejiang) et obtint son titre de docteur en 1562. Il occupa le poste de grand coordinateur du Fujian de 1592 à 1594. Auparavant, il avait travaillé au Guangdong où il avait participé à la lutte contre le banditisme et les wokou. Il fut ensuite envoyé au Fujian. Nous ne connaissons pas la fonction qu’il y occupa lors de ce premier séjour (Ming shi : vol. $24, j .283$, p. 7285 ).

74. Ming shilu leizuan, Fujian-Tuivan juan : 543 : d’après un mémoire du grand coordinateur du Fujian. Zhu Yunchang $x^{2} 3$ 望

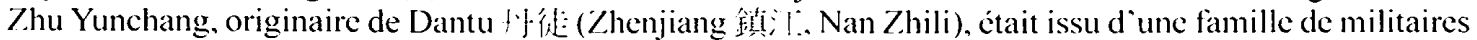
enregistrée dans la garnison de devant de Yunnan, et obtint son titre de docteur en 1580. Avant d'être nommé à cette charge, il avait élé vice-commissaire de gauche des affaires civiles et financières du Fujian.

75. Ba Min tongzhi $1988: j .12$, p. 157. La population fut évacućc à Dajin 人 S: (Funing) en 1388.

76. Il s'agit de groupes de responsabilité fiscale, pouvant constituer à eux seuls des villages, comprenant chacun 110 familles. 
animaux » à partir de $1387^{77}$. Ses habitants auraient disposé de trois jours à peine pour la quitter ${ }^{78}$. Il fallut attendre le règne Wanli (1573-1620) pour qu'elle fut à nouveau exploitée, sous l'impulsion du grand coordinateur Xu Fuyuan, comme nous l'avons vu ci-dessus. C'est à la suite des résultats encourageants obtenus à Haitan qu'il aurait suggéré d'étendre l'opération à l'île de Nanri, à celles du Zhejiang et aux Penghu. Toujours dans le même district de Fuqing, un sort non moins enviable frappa aussi l'île de Xiaolian 小練Il| qui devait être un important centre portuaire d'après la renommée de "petite Yangzhou 小揚州》 qu'elle avait acquise ${ }^{79}$. Celles de Dadeng 大登嶼, de Xiaodeng 小登嶼 et de Gulang 鼓浪嶼, rattachées au district de Tong'an (Quanzhou) et évacuées à la même époque, s'étaient repeuplées à partir de $1470^{80}$. Nan'ao, au Guangdong, connut le même sort et la population dut l'abandonner une première fois en 1387, puis à nouveau en 1409 , après un bref retour en 1404 .

Au début des Qing, l'évacuation de la population côtière vers l'intérieur des terres avait, tout naturellement, concerné aussi les insulaires. Néanmoins, dès 1661, au moment même de la mise en place de cette mesure, la cour avait été contrainte d'édicter un règlement interdisant aux fonctionnaires et aux troupes de faire du commerce, de bâtir des habitations et de développer l'agriculture dans les zones évacuées ${ }^{81}$. En 1672, un édit rappelait les peines dont étaient passibles les membres des administrations locales s'adonnant sans autorisation au cabotage et au négoce outre-mer, ainsi qu'à la mise en culture des îles ${ }^{82}$. Cela montre qu'en dépit de la frilosité de la gestion du gouvernement, une partie d'entre elles étaient probablement déjà bien enracinées dans l'économie du littoral. Après les conflits de la transition dynastique Ming-Qing, certaines d'entre elles purent être repeuplées, mais elles furent soumises, du moins en théorie, à une stricte surveillance. L'établissement de nouveaux habitants y était très contrôlé. En 1801, après un siècle d'essor maritime, une loi restreignant leur développement fut encore promulguée sous la pression des pirates; elle y interdisait toute construction de maison en dur et l'installation de nouveaux venus, en dehors des pêcheurs qui pouvaient continuer à s'y rendre pendant leur activité saisonnière et à y installer leurs ateliers de transformation ${ }^{83}$. En 1790, le gouvernement avait déjà fait procéder à leur inspection afin d'y recenser la population et détruire les constructions illégales ${ }^{84}$.

77. Ba Min tongzhi $: j .5$, p. 70 . À partir de 1949 , avec le repli des troupes nationalistes à Taiwan, de nombreuses îles ont été évacuées et transformées en bases militaires. Haitan n'a été rendue à la population civile et aux touristes qu'en 1992.

78. Fujian tongzhi, éd. Guangxu :j. 86 et 219.

79. Ba Min tongzhi:j. 5, p. 70 .

80. Xiamen zhi $1838: j .2$, p. 42. D’autres îles ont pu être concernées par cette politique, mais les sources ne fournissent les données que pour les plus importantes.

81. Da Qing huidian, éd. Yongzheng : j. 139, p. 2 a.

82. Da Qing huidian, éd. Yongzheng :j. 139, p. 2a-3a.

83. DQHDSL, éd. Guangxu : j.630, p. 5b ; Shanglu dang .上:諭償 (Édits), règne Qianlong : entrée datée du 12/10/1747 (9“ jour du $9^{\circ}$ mois de la $55^{\circ}$ année du règne Qianlong).

84. Gongzhong zhupi zouzhe, junwu-fangwu, 32 : mémoire du gouverneur général du Min-Zhe, Ulana 任拉納, daté du 09/10/1790 (2‘ jour du $11^{\circ}$ mois de la $4^{\circ}$ année du règne Yongzheng).

Ulana était membre du clan Gioro 學羅 et de la bannière rouge unie. Il travailla au Fujian d'abord en qualité de trésorier provincial de 1785 à 1788, puis comme gouverneur du Min-Zhe de 1789 à 1795 . 
Ainsi, les îles furent loin de constituer un élément actif dans le dispositif de défense mis en place par les Qing, même si les plus importantes d'entre elles avaient été investies par des unités militaires. La présence des hommes d'armes n'avait d'ailleurs pas permis d'enrayer une certaine tendance de ces régions à se développer à l'écart du strict contrôle du gouvernement. Dans les années 1830, Nan'ao se présentait toujours comme un centre interlope, aux dires de Giuseppe Maria Callery (1810-1862), alors membre de la Société des missions étrangères :

C'est une grande île placée par $23^{\circ} 30^{\prime}$ de latitude nord, laquelle sert de limites aux provinces de Canton et de Fo-kien, et en même temps de rendez-vous aux navires qui font le commerce d'opium sur les côtes. Un coup de canon ayant annoncé notre arrivée aux navires qui font continuellement station dans cette baie et qui sont comme des magasins d'opium fixes, les capitaines vinrent nous voir au milieu de la nuit à l'endroit où nous avions jeté l'ancre. [...]

Le lendemain j'ai été bien surpris de voir la manière dont se fait le commerce d'opium dans cet endroit ; je m'étais imaginé jusqu'alors que les ténèbres de la nuit n'étaient pas assez épaisses aux yeux des contrebandiers pour cacher leur œuvre d'iniquité ; quel n'a pas été mon étonnement de voir en plein midi une foule de barques pleines de Chinois venir à bord des navires européens el s'en retourner chargées d'opium en vue d'une jonque mandarine qui était à l'ancre tout près de là ! (Callery $1992: 406$ )

Il en fut également ainsi à Taiwan, où dès la réouverture du commerce en 1684 jusqu'à la fin du XVIII' siècle, le convoyage des clandestins vers l'île était, par exemple, devenu une affaire très profitable, voire parfois criminelle avec l'abandon des candidats au milieu du détroit et le développement d'une forme de migration féminine assimilable à une «traite des femmes $»{ }^{85}$. La contrebande de grains à destination du continent fut une autre des activités lucratives qui s'étaient développées à la suite de son rattachement au Fujian et dans laquelle des officiers étaient également compromis ${ }^{86}$. Révoltes et désordres ponctuèrent son rattachement à la Chine, signe d'une faiblesse de sa gestion. Au cours des années 1800 , elle semblait encore terre à conquérir pour le pirate Cai Qian, même si, en définitive, ses tentatives d'installation d'un régime dissident échouèrent - notam-

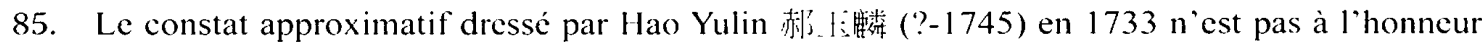
de l'administration côtière : sculs $10 \%$ des candidats clandestins à la traverséc étaient capturés, 20 à $30 \%$ parvenaient à bon port, tandis que 40 à $50 \%$ étaient abandonnés sur des îlots déserts et des bancs de sable sur lesquels ils mourraient de faim ou étaient jetés « en pâture aux poissons 》 ( $v u$ fu 傊腹) [Gongzhong dang Yongzheng chao zouzhe : vol. 21, p. 355-356: mémoire du gouverneur général du Fujian, Hao Yulin, daté du 18/05/1733 (5 jour du $4^{\circ}$ mois de la $11^{\circ}$ année du règne Yongzheng) : Ming Qing shiliao, 1987 : série wu, 280-281). Au sujet du commerce des femmes, voir par exemple Qing shilu, Shengzu Ren Huangdi shilu, vol. 6,j. 253, p. 505 : entrée datée du 27/02/1713 (3 jour du $2^{\circ}$ mois de la $52^{\circ}$ année du règne Kangxi).

Hao Yulin (? - 1745) était membre de la bannière chinoise blanche à bordure. Après avoir commencé sa carrière dans les rangs de l'armée, où il occupa la charge de général des forces des bataillons verts au Yunnan et au Guangdong, il la poursuivit à la tête de l'administration civile en qualité de gouverneur

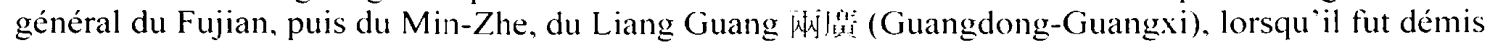
de ses fonctions pour cause de corruption (1741).

86. Voir à ce sujet, $\mathrm{Ng}$ C'hin-keong 1983:113-116. $\dot{\Lambda}$ la page 113 (Gongzhong dang Yongzheng chao zouzhe, vol. 6, p. 523-526), Ng Chin-keong décrit les différentes méthodes employées par les contrebandiers pour convoyer le riz de Taiwan vers le continent, d'après un mémoire de Gao Qizhuo daté du 27/09/1726 (2c jour du 9 mois de la $4^{c}$ année du règne Yongzheng). 
ment grâce au soutien qu'habitants et marchands locaux apportèrent au gouvernement (Calanca 2011: 113-124).

Cet épisode est intéressant car il montre que, malgré une couverture militaire minimale et une présence étatique insuffisante, Taiwan était, grâce à ses réseaux socio-ćconomiques, bien fixée au continent, ou du moins qu'une partie de sa population n'était plus prête à accepter un changement de situation et surtout pas des conduites et des actions pouvant menacer les acquis économiques. Bien que le contexte de la fin du XvIII" siècle et du début $\mathrm{du} X \mathrm{X} \mathrm{X}^{\mathrm{C}}$ ne puisse pas, et sur plusieurs registres, être comparé à celui des années 1720 , il est néanmoins intéressant de se remémorer les dessous de la campagne victorieuse menée par les troupes contre la rébellion de Zhu Yigui. La répression semble en effet avoir bénéficié d'une aide inconditionnelle de la part de certains habitants de Zhangpu (Zhangzhou), officiers travaillant sur le continent ou sur l'île, épaulés par des concitoyens civils qui assumèrent un rôle de conseiller (Salmon 2007 : 237-251). Deux raisons sont susceptibles d'expliquer un tel soutien; elles confirment la place de Taiwan en tant que rempart économique et sécuritaire du Fujian : d'une part, son annexion avait redynamisé l'économie de cette province dans les premières décennies du XVIII ${ }^{\circ}$ siècle en incitant une frange non négligeable de la population des circonscriptions pauvres du littoral méridional, dont des ressortissant de Zhangpu, à y émigrer ; d'autre part, son intégration constituait un garde-fou contre l'établissement de groupements pirates ou de pouvoirs étrangers à même de menacer directement les côtes et la navigation.

Ce dernier exemple montre qu'à la fin de la période retenue, au début du $x^{\prime} x^{c}$ siècle, les îles importantes, en dépit d'un attachement farouche à une certaine forme de liberté, ainsi qu'au relâchement des mœurs et du contrôle étatique, ne pouvaient désormais plus se constituer en «État » dans l'État, comme cela avait pu se produire par le passé, principalement entre le $\mathrm{XVI}^{\mathrm{e}}$ et le début du XVII ${ }^{\mathfrak{e}}$ siècle. Suivant en cela l'évolution du commerce maritime, à partir des années 1680 , le temps n'était plus à l'aventure mais à la consolidation des acquis par l'interpénétration des réseaux économiques chinois et étrangers et ce malgré la politique du gouvernement. Dans les territoires insulaires, l'équilibre des forces s'était établi, beaucoup plus que sous l'impulsion de l'action étatique, au sein même de la population, comme cela avait déjà été le cas parmi la société navigante.

La volonté de la cour de limiter le plus possible l'essor insulaire montre que l'élargissement du périmètre marin reposait encore sur des bases fragiles et ce mème s'il semblait désormais établi que ces endroits constituaient, du moins en théorie, des « barrières naturelles » pour le littoral. Cette vérité était beaucoup plus enracinée chez les ressortissants de la côte qui devaient être plusieurs à considérer, à la suite de Lan Dingyuan, " que ces zones étaient intimement liées au continent, comme les dents l'étaient aux lèvres et ne pouvaient pas être considérées comme des déserts lointains » (Salmon 2007 : 246). Pour beaucoup de fonctionnaires, en particulier les civils, dont l'affectation était régie par la règle de l'incompatibilité (huibi 迥避) qui leur interdisait de travailler dans leur province natale, la réalité des îles leur échappait, car trop éloignée de leur univers. Cela s'appliquait même à celles qui, proches du continent telle Nan'ao, étaient visibles depuis la terre ferme et d'un accès facile et que d'aucuns situaient encore en « haute mer » (waihai), voire dans la «mer extérieure » (haiwai). Ce qui laisse penser que l'appréciation de ces vocables ne sous-entendait pas seulement la distance qui les sépare des rivages, mais qu'il faut probablement y associer d'autres facteurs plus difficile à évaluer. Ils suggèrent, de toute 
évidence, la fragilité de leur localisation aux confins de deux mondes - une mer connue et l'autre inconnue - et, indirectement, la difficulté d'y exercer un pouvoir souverain. La notion de "mer extérieure » (haiwai) évoque l' « impondérable » et peut se rapporter, du moins dans l'imaginaire de certains auteurs, à la masse d'eau aux limites indéfinies qui entourait leur écoumène. Comment d'ailleurs prétendre contrôler ces localités « isolées dans la mer extérieure », lorsque certains fonctionnaires laissaient entendre que leur juridiction était déjà trop vaste pour que l'ensemble des mouillages soit surveillé ${ }^{87}$ ? Le gouvernement a-t-il jamais caressé « l'espoir de venir à bout des contrebandiers ou de la carambouille " ? Celle-ci est, comme le soulignait il y a quelques années Predrag Matvejevitch, "plus qu'un simple savoir-faire, une des sciences de la Méditerranée, ou même son art » (Matvejevitch 1995: 24-25). Des propos que nous pourrions étendre sans difficulté aux régions orientales et méridionales du littoral chinois.

Il aurait été intéressant, pour finir, de savoir si sur les cartes rédigées à la demande du gouvernement au début du $X V \| I^{\mathbb{C}}$ siècle, une limite avait été tracée entre la mer intérieure (neihai) et la haute mer (waihai) et si les îles y avaient été incluses et de quelle façon. Au début des Qing, les plus importantes d'entre elles - les Pescadores, Taiwan et Hainan avaient été intégrées aux cartes, mais souvent en fin de rouleau ou d'ouvrage. Elles étaient transposées en dehors de leur position réelle, masquant ainsi volontairement ou non les voies y conduisant. La localisation de nombreux toponymes insulaires anciens demeure encore aujourd'hui difficile, car s'ils sont cités dans les mémoires au trône ou dans d'autres documents, ils ne figurent, pour beaucoup, sur aucune des projections de la côte à notre disposition. Ces omissions relèvent-elles d'une volonté stratégique désireuse de cacher leur emplacement pour des raisons de sécurité, ou plus simplement de l'isolement dans lequel le gouvernement souhaita les laisser jusqu'au moins la fin du XviII ${ }^{\circledR}$ siècle, quand il fut contraint de procéder au recensement de leur population sous la pression des pirates ? Foyers possibles de rébellion au pouvoir central, certaines îles montrèrent effectivement qu'elles pouvaient se mesurer au continent, d'où certainement la frilosité des autorités à leur égard. Il reste néanmoins à noter que, envers et contre la volonté du gouvernement, voire des territoires insulaires eux-mêmes, elles jouèrent souvent un rôle décisif dans le processus d'élargissement de l'espace maritime chinois.

\section{BIBLIOGRAPHIE}

\section{Sources primaires et compilations}

Ba Min tongzhi 八閩通志 (Histoire générale du pays de Min, 1491), Huang Zhongzhao 黄仲牟 (comp.), Pékin, Shumu wenxian chubanshe, Beijing tushuguan guji zhenben congkan 33-34, 1988.

CHFN Renxi 陳仁錫

1965 Huang Ming shifa lu 是朾世法錄 (Recueil institutionnel, 1630), Taipei, Zhongguo shixue congshu.

Chouhai tubian 等海圖捔 (Précis de défense maritime, 1561-1562), Zheng Ruozeng 鄭|'若曾 (comp.), Pékin, Zhonghua shuju, 2007.

Da Qing huidian 大清曾典l (Statuts de la dynastie Qing, 1733), éd. Yongzheng.

87. Gao Qizhuo fait ainsi remarquer qu'étant donné le grand nombre d’îles et d'îlots disséminés le long du littoral, il n'est pas possible d'arrêter tous les pirates (Gongzhong dang Yongzheng chao zouzhe : vol. 6, p. 684-686). 
$D Q H D S L$, éd. Guangxu

1908 (Qinding) Da Qing huidian shili (欽定) 大清會典事例 (Statuts et précédents de la dynastie Qing, édités par volonté impériale, 1899), Li Hongzhang 李鴻草 (comp.) et al., Shanghai, Shangwu yinshuguan.

Erya zhushu 爾雅注䟽 (Commentaires au Erya), Xing Bing 所呙 (comp.), Pékin, Beijing Daxue chubanshe, «Shisan jing zhushu », vol. 24, 2000.

Fujian tongzhi 福建通志 (Histoire générale du Fujian), éd. Guangxu.

Gongzhong dang Yongzheng chao zouzhe 宮中檔雍泟朝奏摺 (Mémoire au palais du règne Yongzheng), Taipei, Gugong bowuyuan, 1977-1980.

Kangxi chao hanwen zhupi zouzhe huibian 康熙朝漢文硃批奏摺氣編 (Recueil de mémoires au palais du règne Kangxi), Zhongguo diyi lishi dang'anguan 中國第一 歷史檔案館 (comp.), Pékin, Dang'an chubanshe, 1984.

Kangxi tongyi Taiwan dang 'an shiliao xuanji 捸熙統一臺灣檔案这料選輯 (Sélection de documents d'archives relatifs à l'annexion de Taiwan sous le règne Kangxi), Xiamen Daxue Taiwan yanjiusuo 廈門大學臺灣研究所 et Zhongguo diyi lishi dang'anguan bianjibu 中國第一歷史檔案館編輯部' (comp.), Fuzhou, Fujian renmin chubanshe, Qingdai Taiwan dang'an shiliao congkan, 1983.

LAN Dingyuan 藍鼎元

1730 Luzhou chuji 鹿洲初集 (Premier recueil des œuvres de Luzhou [Lan Dingyuan]).

Ming Qing shiliao 师清史料 (Documents historiques des dynasties Ming et Qing), Pékin, Zhonghua shuju, 1987.

Ming shi 明史 (Histoire de la dynastie Ming), Zhang Tingyu 張廷洰 (comp.) et al., Pékin, Zhonghua shuju, 1987.

Ming shilu leizuan, Fujian-Taiwan juan 明實錄類纂：福建臺灣卷 (Recueil thématique des Chroniques véridiques de la dynastie Ming, volume [consacré] au Fujian et à Taiwan), Xue Guozhong 薛國忠 (comp.), Wei Hong 韋洪, Li Guoxiang 李國祥 \& Yang Chang 楊昶 (eds.), Wuhan, Wuhan chubanshe, 1993.

Nan'ao zhi 南澳志 (Monographie de l'île de Nan'ao), éd. Qianlong.

Qing jingshi wenbian 清經世文編 (Recueil de textes sur l'administration de la dynastie Qing, 1836), He Changling 賀長齡 \& Wei Yuan 魏源 (comp.), Pékin, Zhonghua shuju, 1992.

Qing shi gao 清史稿 (Ébauche d'histoire Qing, 1927), Zhao Erxun 趙爾巽 (comp.) et al., Pékin, Zhonghua shuju 1976-1977.

Qing shi liezhuan 清史列傳 (Biographies de l'histoire de la dynastie Qing, 1928), Wang Zhonghan 王鐘翰 (comp.), Pékin, Zhonghua shuju, 1987.

Qing shilu, Shengzu Ren Huangdi shilu 清實錄聖祖仁皇帝實錄 (Annales véridiques de l'empereur Shengzu), Pékin, Zhonghua shuju, 1985.

Qing shilu, Shizu Zhang Huangdi shilu 清實錄世祖章皇帝實錄 (Chroniques véridiques de l'empereur Shizu), Pékin, Zhonghua shuju, 1985.

San guo zhi 三國志 (Histoire des Trois Royaumes), Chen Shou 陳壽 (comp.), «Wei zhi » 魏志 (Histoire de Wei), Taipei, Commercial Press, 1976. 
Shi ming 釋名, comp. Liu Xi 劉熙, éd. du Sibu congkan 四部叢刊 (Collection suivant les quatre catégories, 1919-1936), Shanghai, Commercial Press, 1989.

Shuowen jiezi 說文解 (Dictionnaire étymologique des caractères), Pékin, Zhonghua shuju, 1998.

Song shi 宋史 (Histoire de la dynastie Song, 1345), Tuotuo (Toghto) 脫脫 (éd.), Pékin, Zhonghua shuju, 1977.

(Chongxiu) Taiwan fuzhi (重修) 臺灣府志 (Monographie de la préfecture de Taiwan, 1748), Fan Xian 範咸 (rév.) et al., Pékin, Zhonghua shuju, 1985.

Xiamen zhi 廈門志 (Monographie de Xiamen, 1838), Zhou Gai 周凱 (rév.) et al., Taipei, Chengwen chubanshe, Zhongguo fangzhi congshu, Huanan difang 80,1967.

Zhejiang tongzhi 浙汇通志 (Histoire générale de la province du Zhejiang, 1736), Ji Zengyun 稽曾筠 \& Li Wei 李衛 (rév.), Shanghai, Shanghai guji chubanshe, 1991.

Zhoushan zhi 舟ll志 (Monographie de Zhoushan, Ming), He Rubin 何汝賓 (comp.), Taipei, Chengwen chubanshe, 1983.

Zhufan zhi zhubu 諸蕃志注補 (Traduction annotée de la Relation sur les pays étrangers), Han Zhenhua 斡振華 (comp.), Hong Kong, Centre of Asian Studies Occasional Papers and Monographs, 2000.

Wenyuan ge Siku quanshu [Bibliothèque complète en quatre sections, édition Wenyuan ge (Bibliothèque du palais)], Taipei, Commercial Press, 1983.

\section{Sources secondaires}

\section{$D M B$}

1976 Goodrich Luther Carrington and Fang Chaoying, Dictionary of Ming Biography, 1368-1644, New York, Columbia University Press.

\section{$E C C P$}

1943 Arthur W. Hummel, Eminent Chinese of the Ch'ing Period (1644-1912), Washington, United States Government Printing Office.

Buussé, Leonard

1990 "Minnan-jen or cosmopolitan? The rise of Cheng Chih-lung alias Nicolas Iquan", in Eduard B. Vermeer (ed.), Development and Decline of Fukien Province in the 17th and 18th Centuries, Leiden, Brill, Sinica Leidensia 23, p. 245-264.

Bors, Jean-Pierre

2002 "Principes tactiques de la défense littorale au XVII" siècle », in Jean-Pierre Bois (éd.), Défense des côtes et cartographie historique, Paris, éditions du CTHS, p. 53-65.

Calanca, Paola

2001 «Aspects spécifiques de la piraterie à Hainan sous les Ming et au début des Qing », in Roderich Ptak \& Claudine Salmon (eds.), Hainan. De la Chine à l'Asie du Sud-Est, Wiesbaden, Harrassowitz Verlag.

2007 《Fujian shuishi jiangling : yizhi jingying zhi shi ? 》福建炎师将领：- - 支“ 精英之师”? (Les officiers navals du Fujian, un 'corps d'élite' ?), in Faguo hanxue 法國漢學 12 (Sinologie française), Zhongguo bianchen jiangli 中國邊臣疆吏 (Les fonctionnaires des frontières), Paola Calanca, Fabienne Jagou \& Li Guoqiang (eds.), Zhonghua shuju, p. 86-101. 
2011 Piraterie et contrebande au Fujian. L'administration chinoise face aux problèmes d'illégalité maritime ( $x^{V} I I^{\prime}$-début $x I x^{e}$ siècle), Paris, Les Indes savantes.

Callekr, Joseph-Marie

1992 Voyage sur les côtes de Chine fait en 1838, extrait des Nouvelles Annales des voyages, in Muriel Détrie \& Ninette Boothroyd (eds.), Le voyage en Chine. Anthologie des voyageurs occidentaux du Moyen Âge à la chute de l'empire chinois, Paris, Robert Laffont, Bouquins.

CHFN Shangsheng 陳尚勝

1993 Kaifang yu biguan. Zhongguo fengjian wanqi duiwai guanxi yanjiu 開放與 閉關. 中國封建晚期對外關係研究 (Ouverture et fermeture des frontières. Une étude des relations chinoises avec l'étranger à la fin de l'époque féodale), Jinan, Shandong renmin chubanshe.

CHEN Wenshi 陳文石

1966 Ming Hongwu-Jiajing jian de haijin zhengce 明洪武嘉靖間的海禁政策 (La politique de prohibition maritime du règne Hongwu au règne Jiajing), Taipei, Guoli Taiwan Daxue, Wenli congkan 20.

Church, Sally

2006 "Conception of Maritime Space in Xu Jing's Xuande fengshi Gaoli tujing", in Angela Schottenhammer \& Roderich Ptak (eds.), p. 79-107.

DARS, Jacques

1992 La marine chinoise du xe siècle au XIV siècle, Paris, Economica, études d'histoire maritime 11 .

DENG Yanlin 鄧衍林

1958 Zhongguo bianjiang tuji $l u$ 中國邊疆圖籍錄 (Catalogue des ouvrages relatifs aux frontières chinoises), Shanghai, Shangwu yinshuguan.

FAIRBANK, John

1969 Trade and Diplomacy on the China Coast: The Opening of the Treaty Ports, 1842-1854, Stanford, Stanford University Press.

Fuchs, Walter

1946 "The 'Mongol Atlas' of China by Chu Ssu-pen and the Kuangyu t'u", Monumenta Serica, Monograph series 8, Pei-ping.

HIRTH, Friedrich \& RockhiLl, William Woodville

1967 Chau-Ju-Kua: His work on the Chinese and Arab Trade in the twelfth and thirteenth Centuries, Cheng-Wen Publishing Company.

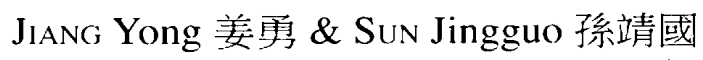

2011 《Fujian haifang tu chutan》福建海防圖初探 (Début de l'analyse de la Carte de la défense maritime du Fujian), Gugong bowuyuan yuankan 故宮博物院 院刊 1, p. 67-72).

LANCIOTTI, Lionello

1995 “Il 'mare' nelle antiche fonti letterarie cinesi”, Cina 25, p. 7-13.

Li Deyuan 李德元

2006 Ming Qing shiqi hainei yimin yu haidao kaifa 明清時期海内移民與海島開發 (Migrations et exploitation insulaire à l'époque Ming et Qing), Xiamen, Xiamen daxue chubanshe. 
LI Sheng 厲聲 \& Li Guoqiang 李國強

2002 Zhongguo bianjiang shi di yanjiu congshu, 1989-1998 中國邊疆史地研 究綜述 (État de la recherche historique et géographique sur les frontières chinoises de 1989 à 1998), Harbin, Heilongjiang jiaoyu chubanshe.

Lı Xiaocong 李孝㙂

2006 Zhongguo lishi shang haiyang kongjian yu yanhai di tu 中國歷史上海洋 空間與沿海地圖 (Historique de l'espace maritime et des cartes du littoral chinois), in Angela Schottenhammer \& Roderich Ptak (eds.), p. 155-176.

Matvejevitch, Predrag

1995 Bréviaire méditerranéen, Paris, Payot \& Rivages.

Michelet, Jules

1861 La mer, Paris, Hachette.

Mills, J. V.

1954 "Chinese Coastal Maps", Imago Mundi 11, p. 151-168.

Moll-Murata, Christine

2006 "Sundry Notes on the Zhoushan Archipelago: Topographical Notation and Comparison to the Braudelian Islands", in Angela Shottenhammer \& Roderich Ptak (eds.), p. 109-123.

Netol.IT7.KY, Almut

1977 Das Ling-wai tai-ta von Chou Ch'ü-fei, Eine landeskunde Sïdchinas aus dem 12. Jahrhundert, Wiesbaden, Franz Steiner Verlag GMBH, Münchener ostasiatische Studien, Band 21.

$\mathrm{NG}$ Chin-keong

1997 "Maritime Frontiers, territorial Expansion and Hai-fang", in Sabine Dabringhaus \& Roderich Ptak (eds.), China and Her Neighbours. Borders, Visions of the Other; Foreign Policy, 10th to 19th Century, Harrassowitz Verlag, Wiesbaden.

OUYANG Zongshu 歐陽宗書

1998 Haishang renjia. Haiyang yuye jingji yu yumin shehui 海1: 人家 - 海洋漁業 經濟與渙民社會 (Les hommes de la mer. Économie et société halieutique), Nanchang, Jiangxi gaoxiao chubanshe, Haiyang yu Zhongguo congshu.

SALMON, Claudine

2006 "Coastal Maps from the Beginning of the Qing Dynasty, with special reference to the Qingchu haijiang tushuo", in Angela Schottenhammer \& Roderich Ptak (eds.), p. 177-189.

2007 «Cong Zhu Yigui qishi de zhenya shiyi (1721) laikan Zhangpu ren zhi wangluo 》從来一貴起事的鎮厴事宜來看漳浦人之網終 (Le réseau de Zhangpu vu à travers la répression de la rébellion de Zhu Yigui, 1721), in Faguo hanxue 12 (Sinologie française), Zhongguo bianchen jiangli 中國邊 臣疆吏 (Les fonctionnaires des frontières), Paola Calanca, Fabienne Jagou \& Li Guoqiang (eds.), Zhonghua shuju, p. 237-251.

Schottenhammer, Angela \& PTak, Roderich (eds.)

2006 The perception of Maritime Space in Traditional Chinese Sources, Wiesbaden, Harrassowitz Verlag. 
Sorg Zhenghai 宋正海 Guo Yonglao 郆永芳 \& CHEN Ruiping 陳瑞平

1989 Zhongguo gudai haiyang xueshi 中國吉代海洋學史 (Histoire de l'océanographie chinoise ancienne), Pékin, Haiyang chubanshe.

Sun Guangqi 孫光圻

1989 Zhongguo gudai hanghai shi 中國古代航海史 (Histoire de la navigation ancienne chinoise), Pékin, Haiyang chubanshe.

Sin Guangqi (ed.)

2007 Zhongguo hanghai shi jichu wenxian huibian 中国航海史基础文献汇编 (Recueil des sources sur l'histoire de la navigation chinoise), Pékin, Haiyang chubanshe.

WALEY, Arthur

1959 The Opium War through Chinese Eyes, New York, The Macmillan Company.

Wavg Hongbin 王宏斌

2002 Qingdai qianqi haifang : sixiang yu zhidu 清代解期海防：思想與制度 (Conception et organisation de la défense maritime au début des Qing), Pékin, Shehui kexue wenxian chubanshe, Dongfang lishi xueshu wenku.

WARD FAY, Peter

1975 The Opium War, 1840-1842, Chapel Hill, University of North Carolina Press.

WIETHOFF, Bodo

1969 China dritte Grenze. Der traditionelle chinesische Staat und der küstennahe Seeraum, Wiesbaden, Otto Harrassowitz.

Xu Chonghao 許崇影

1975 Qiongya zhilüe 瓊崖志略 (Géographie de Hainan, 1945), Taipei, Taiwan xuesheng shuju (rpt).

Z HANG Xun 章巽 (ed.)

1991 Zhongguo hanghai keji shi 中國航海科技史 (Histoire de la technique navale chinoise), Pékin, Haiyang chubanshe.

ZHU Weigan 朱維幹

1986 Fujian shigao 福建史稿 (Ébauche pour une histoire du Fujian), Fu'an, Fujian jiaoyu chubanshe.

Zov Keyuan

2005 Law of the Sea in East Asia. Issues and prospects, London \& New York, Routledge Studies in International law, Routledge Curzon. 


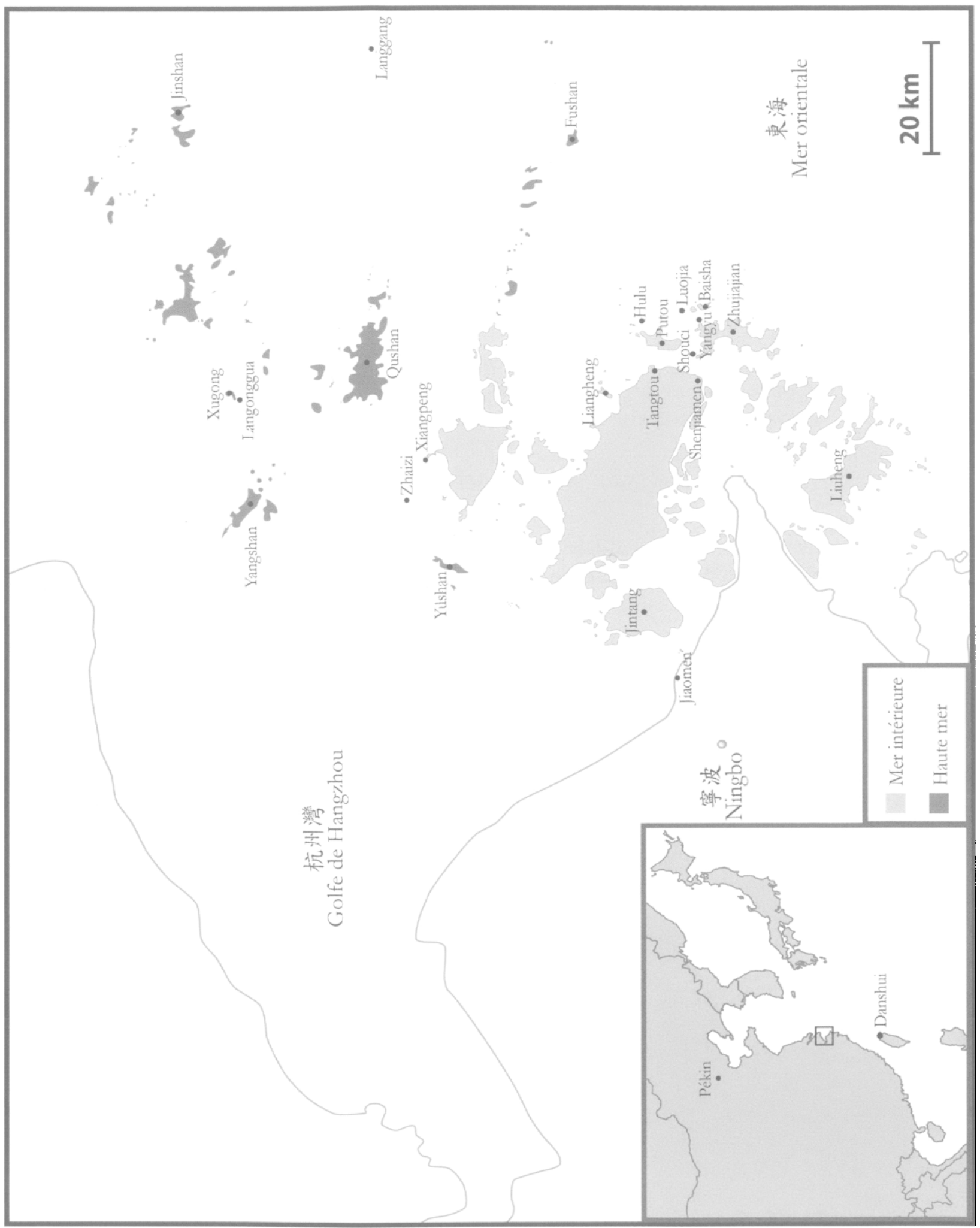

Limites entre mer intérieure et haute mer (district de Dinghai, xvıı` siècle) (Paola Calanca, Wu Chun-huei). 